\title{
On Soliton-type Solutions of Equations Associated with
}

\author{
N-component Systems *
}

\author{
Mark S. Alber ${ }^{\dagger}$ \\ Department of Mathematics \\ University of Notre Dame \\ Notre Dame, IN 46556 \\ Mark.S.Alber.1@nd.edu \\ Gregory G. Luther $\ddagger$ \\ Engineering Sciences and Applied Mathematics Department \\ McCormick School of Engineering and Applied Science \\ Northwestern University \\ 2145 Sheridan Road \\ Evanston, Il 60208-3125 \\ ggluther@nwu.edu \\ Charles Miller \\ Department of Mathematics \\ University of Notre Dame \\ Notre Dame, IN 46556 \\ cmiller6@nd.edu
}


November 18, 2018

byline: Soliton-type Solutions and $N$-component Systems

\begin{abstract}
The algebraic geometric approach to $N$-component systems of nonlinear integrable PDE's is used to obtain and analyze explicit solutions of the coupled KdV and Dym equations. Detailed analysis of soliton fission, kink to anti-kink transitions and multi-peaked soliton solutions is carried out. Transformations are used to connect these solutions to several other equations that model physical phenomena in fluid dynamics and nonlinear optics.
\end{abstract}

\title{
1 Introduction
}

The solution of nonlinear evolution equations using techniques from algebraic geometry was initially developed to handle $N$-phase wave trains. With this approach, parameterized families of quasi-periodic and soliton solutions are associated with Hamiltonian flows on level sets of finitedimensional phase spaces. In Section 2, these flows are described using the $\mu$ variable representation on symmetric products of Riemann surfaces. The first integrals in the quasi-periodic case have the form $P_{j}^{2}=C\left(\mu_{j}\right)$ where $C\left(\mu_{j}\right)$ is a polynomial with constant coefficients and $P_{j}$ is the conjugate variable for $\mu_{j}$. The polynomial $C(E)$ is called the spectral polynomial and determines the form of the first integrals.

The algebraic geometric approach provides a way to construct solutions by analytical or numerical integration of a system of Hamiltonian equations for these $\mu$ variables. Solutions of the

${ }^{*}$ PACS numbers 03.40.Gc, 11.10.Ef, 68.10.-m, AMS Subject Classification 58F07, 70H99, 76B15

${ }^{\dagger}$ Research partially supported by NSF grant DMS 9626672.

${ }^{\ddagger}$ Research partially supported by NSF grant DMS 9626672. 
nonlinear PDE's are expressed in terms of these variables by using the trace formulas. The $\mu$ variable representation yields an action angle representation on a Jacobi variety (invariant variety in the phase space) that linearizes the Hamiltonian flow, and in the quasi-periodic case the solution of the $\mu$ equations is reduced to a Jacobi inversion problem. Solutions are then expressed using Riemann theta functions. For details see, for example, Mumford(1983) and Ercolani and McKean (1990).

As pairs of roots of the polynomial $C(E)$ coalesce, soliton solutions begin to appear. Applying this limit to the first integrals and the equations of motion in terms of the $\mu$ variables for quasiperiodic solutions, Hamiltonian systems of ODE's that describe soliton solutions are obtained. Soliton solutions are computed by solving these equations and using the trace formula to connect them to the associated nonlinear PDE's. In the soliton limit the Jacobi inversion problem for the system often reduces to a system of algebraic equations. As will be shown, these algebraic equations are exactly solvable in the case of genus one and two. For details about the connection between soliton and quasi-periodic solutions see for example Ablowitz and Ma (1981) and Alber and Alber(1985). The exact representations obtained for soliton solutions are shown to be related to the Hirota $\tau$-functions. The case of genus $n$ solutions is analogous to the lower genus case and can be described using similar formulas. Using the action-angle representation, the algebraic geometric approach also introduces a powerful way to compute the phase shifts due to soliton interactions (see Alber and Marsden(1992) for details.)

In this paper, soliton solutions of multicomponent systems of equations are studied using the algebraic geometric approach. The soliton fission effect, kink to anti-kink transitions, and multipeaked solitons are demonstrated using a class of commuting Hamiltonian systems on Riemann surfaces. These first two effects manifest themselves in the soliton limit of the genus two quasiperiodic solution when six roots of the spectral polynomial $C(E)$ coalesce in a pairwise fashion. 
Exact formulas for these solutions are obtained and asymptotic and numerical analysis of them is performed. The technique used to obtain these limiting solutions is demonstrated explicitly in the case of the coupled Korteweg-de Vries (cKdV) and the coupled Dym (cDym) equations and physically relevant equations associated with them.

The modified cKdV system is

$$
\begin{aligned}
& u_{t}=v_{x}-\frac{3}{2} u u_{x}+K_{1} u_{x}, \\
& v_{t}=\frac{1}{4} u_{x x x}-v u_{x}-\frac{1}{2} u v_{x}+K_{1} v_{x}
\end{aligned}
$$

which reduces to the cKdV system for $K_{1}=0$. Otherwise $K_{1}$ is viewed as a small constant. The coupled Dym equations are given by

$$
\begin{aligned}
& u_{t}=\frac{1}{4} u_{x x x}-\frac{3}{2} u u_{x}+v_{x}+K_{1} u_{x}, \\
& v_{t}=-u_{x} v-\frac{1}{2} u v_{x}+K_{1} v_{x} .
\end{aligned}
$$

The general method is demonstrated by describing quasi-periodic and soliton solutions for the cKdV and cDym systems for genus two and less.

The cKdV and cDym equations are both generic examples of $N$-component systems. Energy dependent Schrődinger operators and $b i$-Hamiltonian structures for multicomponent systems were investigated in Antonowicz and Fordy(1987). Quasi-periodic and soliton solutions were studied in connection with Hamiltonian systems on Riemann surfaces in Alber et al.(1997). In Alber et al. (1994) it was also shown that the presence of a pole in the associated Schrödinger operator yields a special class of weak billiard solutions for nonlinear PDE's.

The soliton fission effect, kink to anti-kink transitions, and multi-peaked solitons extend to equations that model physical phenomena. The generalized Kaup equation, the classical Boussinesq system, and the equations governing second harmonic generation (SHG) are each connected to the cKdV system through nonsingular transformations. Direct application of these transformations 
enables solutions of the cKdV system to be interpreted in the context of these related equations. Such transformations are given explicitly in Appendix A. Both the Boussinesq System,

$$
\begin{aligned}
U_{t}+W_{x}+U U_{x} & =\gamma U_{x}, \\
W_{t}+U_{x x x}+(W U)_{x} & =\gamma W_{x},
\end{aligned}
$$

and the generalized Kaup equations

$$
\begin{aligned}
\pi_{t} & =\phi_{x x}+\frac{1}{3}(1-3 \sigma) \delta^{2} \phi_{x x x x}-\epsilon\left(\phi_{x} \pi\right)_{x}+\alpha \pi_{x}, \\
\phi_{t} & =-\alpha \phi_{x}+\frac{\epsilon}{2} \phi_{x}^{2}+\pi,
\end{aligned}
$$

arise from the theory of shallow water waves (see Whitham(1974)). Here $\gamma$ and $\alpha$ are small parameters.

In optics, the interaction of a wave envelope at frequency and wavenumber $(w, k)$ with a second wave at twice the frequency is modeled by the system of equations

$$
\begin{aligned}
& \left(q_{1}\right)_{x}=-2 q_{2} q_{1}^{*}, \\
& \left(q_{2}\right)_{\tau}=q_{1}^{2} .
\end{aligned}
$$

This process is called second harmonic generation in nonlinear optics and is used to convert laser light to its second harmonic frequency.

The scattering problem for the energy dependent Schődinger operators was studied by Jaulent(1972) and Jaulent and Jean(1976). The completely integrable variant of the Boussinesq system (1.5)(1.6) was first introduced by Kaup(1972). In Matveev and Yavor(1979) $\theta$-functions were used to describe quasi-periodic solutions of the Boussinesq System. They also described a particular type of $N$-soliton solution using singular classes of $\theta$-functions. Rational solutions were studied in Sachs(1998) in connection with a pair of Calogero-Moser equations coupled through the constraints. Martinez Alonso and Medina Reus(1992) and Estevez et al.(1994) described some of the 
soliton solutions using Hirota's $\tau$-functions. Using asymptotics of these soliton solutions they also demonstrated soliton fission. A connection between the SHG system and the cKdV system was recently discussed by Khusnutdinova and Steudel(1998).

\section{Generating Equations for the Coupled KdV and Dym Equations}

We begin by describing the general approach of generating equations and applying it to the cKdV and cDym equations. Details for the general case of $N$-component systems are discussed in Alber et al.(1997).

\subsection{Dynamical Generating Equations.}

The hierarchy of the coupled KdV and Dym equations is obtained as the compatibility condition for the eigenfunction of the linear system of equations

$$
\begin{aligned}
L \psi & =0, \\
\psi_{t} & =A \psi .
\end{aligned}
$$

The time flow is produced by the linear differential operator

$$
A=B \frac{d}{d x}-\frac{B_{x}}{2}
$$

where $B(x, t, E)$ is a specified rational function. The operator $L$ is assumed to be of the energy dependent Schrődinger type,

$$
L=-\frac{d^{2}}{d x^{2}}+V(x, t, E)
$$

with a rational potential having the form

$$
V(x, t, E)=\frac{\sum_{j=0}^{N} v_{j}(x, t) E^{j}}{\sum_{i=0}^{M} r_{i} E^{i}},
$$


where $r_{i}$ are constants and $v_{j}(x, t)$ are functions of the variable $x$ and the parameter $t . E$ is a complex spectral parameter. In particular, the potential is chosen as

$$
V(E)=\kappa E^{2}+u(x, t) E+v(x, t)
$$

for the cKdV system or

$$
V(E)=u(x, t)+E+\frac{v(x, t)}{E}
$$

to recover the $\mathrm{cDym}$ system. Here $\kappa= \pm 1$. One chooses $\kappa=-1$ to establish the transformation from the cKdV system to the SHG system, and $\kappa=1$ to establish the transformation from the cKdV system to the Boussinesq System. Notice that the main difference between the cKdV and cDym systems is the presence of a pole in the Schrödinger operator (2.4) associated with the cDym equations. The pole in the potential for the cDym case was shown in Alber et al.(1994) to be a necessary feature for systems with weak billiard solutions.

The compatibility condition of (2.1)-(2.2) can be found by taking the $t$ derivative of (2.1), acting on (2.2) by $L$, and forcing the fact that these two operators commute. This leads to the following system of equations

$$
\left(L_{t}+[L, A]\right) \psi=0, \quad L \psi=0
$$

where $[L, A]=L A-A L$ is the commutator of $L$ and $A$. Using the definition of the differential operator $A$ in (2.3) and $L$ in (2.4), this Lax equation yields

$$
\frac{\partial V}{\partial t}=-\frac{1}{2} \frac{\partial^{3} B}{\partial x^{3}}+2 \frac{\partial B}{\partial x} V+B \frac{\partial V}{\partial x},
$$

which is a generating equation for the coefficients of the differential operator $A$. By taking $B$ to be the rational function,

$$
B(x, t, E)=\sum_{k=-r}^{m} b_{m-k}(x, t) E^{k}=E^{-r} \prod_{k=1}^{n}\left(E-\mu_{k}(x, t)\right),
$$


substituting it into the generating equation (2.9) and equating like powers of $E$, a recurrence chain of equations for the coefficients $b_{j}$ is obtained. Evaluating these equations one by one, a PDE for the coefficient $b_{n}$ is obtained, where $n=m+r$. By considering all possible values of $n$ and $m$, a hierarchy of systems generated by the Lax equation with a given potential (generating equation) is obtained.

Assuming that $\frac{\partial V}{\partial t}=0$ in (2.9) and integrating gives the stationary generating equation which has the form

$$
-B^{\prime \prime} B+\frac{1}{2}\left(B^{\prime}\right)^{2}+2 B^{2} V=C(E)
$$

where the choice of $B(E)$ from (2.10) ensures that $C(E)$ is a rational function with constant coefficients. These coefficients are the first integrals and parameters of the coupled system of equations. (For details about the general method see Alber and Alber(1985).) This equation gives rise to level sets in the phase space $\mathbf{C}^{n}$ corresponding to the Riemann surface

$$
W^{2}=C(E) .
$$

The dynamics of genus $n$ quasi-periodic solutions for $u$ and $v$ with respect to the $x$ and $t$ coordinates are captured as flows on the level set produced by a symmetric product of $n$ copies of the Riemann surface (2.12) (see Alber et al.(1997) for details).

The method of generating equations yields the $\mathrm{cKdV}$ and $\mathrm{cDym}$ equations by using $B(x, t, E)=$ $b_{0}(x, t) E+b_{1}(x, t)$. In Appendix $\mathrm{A}$ solutions of the generalized Boussinesq and generalized Kaup equations are linked to these systems. Further, the second harmonic generation equations are obtained when $B(x, t, E)=b_{2}(x, t) E^{-1}$. 


\section{Solutions of the cKdV and cDym Systems from Dynamical Sys- tems on Riemann Surfaces}

In this section we obtain finite-dimensional Hamiltonian systems on Riemann surfaces for the $\mu$ variables defined in $(2.10)$ as the roots of the function $B(x, t, E)$. These systems capture the essential dynamics of quasi-periodic and soliton solutions of the $\mathrm{cKdV}$ and cDym systems. The quasi-periodic solutions are often called $n$-gap solutions in physics literature and quasi-periodic solutions of genus $n$ in mathematics literature. In Appendix B the solutions of these finite-dimensional Hamiltonian systems are linked to the solutions of the integrable nonlinear PDE's through trace formulas of

the general form $u=\alpha \sum_{j=1}^{n} \mu_{j}+\beta$, where $\alpha$ and $\beta$ are constants. This general construction also provides links between the solutions of the $\mathrm{cKdV}$ and cDym systems and other nonlinear PDE's.

Choose $B(x, t, E)$ as in (2.10) where $n=r+m$ is the genus of the desired solution. For the cKdV case, we substitute (2.6) into (2.9) and (2.11) to obtain

$$
u_{t} E+v_{t}=-\frac{1}{2} B^{\prime \prime \prime}+2 \kappa B^{\prime} E^{2}+2 B^{\prime} u E+2 B^{\prime} v+B u^{\prime} E+B v^{\prime}
$$

and

$$
-B^{\prime \prime} B+\frac{1}{2}\left(B^{\prime}\right)^{2}+2 \kappa B^{2} E^{2}+2 B^{2} u E+2 B^{2} v=C(E) .
$$

For the cDym system we substitute the potential (2.7) into the same equations to obtain

$$
u_{t} E+v_{t}=-\frac{1}{2} B^{\prime \prime \prime} E+2 B^{\prime} E u+2 B^{\prime} E^{2}+2 B^{\prime} v+B u^{\prime} E+B v^{\prime}
$$

and

$$
-B^{\prime \prime} B+\frac{1}{2}\left(B^{\prime}\right)^{2}+2 B^{2} u+2 B^{2} E+\frac{2 B^{2} v}{E}=C(E)
$$

By equating like powers of $E$ on the left and right hand side of the equations (3.2) and (3.4) we 
obtain the necessary forms for $C(E)$. Therefore we write

$$
C(E)=2 \kappa E^{-2 r} \prod_{i=1}^{2(n+1)}\left(E-m_{i}\right)
$$

for $\mathrm{cKdV}$ equations and

$$
C(E)=2 E^{-(2 r+1)} \prod_{i=1}^{2(n+1)}\left(E-m_{i}\right)
$$

for the cDym equations, where the real numbers $m_{i}$ are the roots of the polynomials $C(E)$.

\subsection{Finite-Dimensional Hamiltonian Systems.}

From the generating equation above it follows that the $\mu_{i}$ 's, which are the roots of the function $B(x, t, E)$, are solutions of finite-dimensional Hamiltonian systems. By solving these Hamiltonian systems and using the trace formula, the dynamics of the roots $\mu_{i}$ are connected to the functions $u$ and $v$ to obtain solutions of the PDE's.

Namely, Hamiltonian equations for $\mu_{i}$ 's are obtained by substituting $E=\mu_{i}$ into the generating equations (3.2), (3.4). This yields the following systems of equations for cKdV:

$$
\mu_{i}^{\prime}= \pm \frac{2 \sqrt{\kappa \prod_{j=1}^{2 n+2}\left(\mu_{i}-m_{j}\right)}}{\prod_{j \neq i}\left(\mu_{i}-\mu_{j}\right)} \quad i=1, \ldots, n
$$

for the flow in space, and

$$
\dot{\mu}_{i}=\mp \frac{2\left(\sum_{j \neq i} \mu_{j}\right) \sqrt{\kappa \prod_{j=1}^{2 n+2}\left(\mu_{i}-m_{j}\right)}}{\prod_{j \neq i}\left(\mu_{i}-\mu_{j}\right)} \quad i=1, \ldots, n,
$$

for the flow in time. Here $n=m+r$ from the definition of $B$ in (2.10) and $m_{i}$ are fixed real roots of $C(E)$ in (3.5). The plus/minus refers to which branch of the Riemann surface (2.12) the solution is on. Systems (3.7)-(3.8) are commuting Hamiltonian systems with Hamiltonians

$$
H=\sum_{j=1}^{n} \frac{D\left(\mu_{j}\right)\left(P_{j}^{2}-C\left(\mu_{j}\right)\right)}{\prod_{r \neq j}^{n}\left(\mu_{j}-\mu_{r}\right)}
$$


where $D\left(\mu_{i}\right)=1$ and $D\left(\mu_{i}\right)=-\sum_{j \neq i} \mu_{j}$ in the stationary and dynamical cases respectively. These systems share the same complete set of first integrals: $P_{j}^{2}=C\left(\mu_{j}\right), j=1, \ldots, n$ where the polynomial $C(E)$ is defined by (3.5) (for details see Alber et al.(1997)).

For the cDym equation we see that

$$
\mu_{i}^{\prime}= \pm \frac{2 \sqrt{\prod_{j=1}^{2 n+2}\left(\mu_{i}-m_{j}\right)}}{\sqrt{\mu_{i}} \prod_{j \neq i}\left(\mu_{i}-\mu_{j}\right)} \quad i=1, \ldots, n
$$

for the spatial flow, and

$$
\dot{\mu}_{i}=\mp \frac{2\left(\sum_{j \neq i} \mu_{j}\right) \sqrt{\prod_{j=1}^{2 n+2}\left(\mu_{i}-m_{j}\right)}}{\sqrt{\mu_{i}} \prod_{j \neq i}\left(\mu_{i}-\mu_{j}\right)} \quad i=1, \ldots, n
$$

for the time flow. Systems (3.10)-(3.11) are also Hamiltonian systems and they share the same complete set of first integrals: $P_{j}^{2}=C\left(\mu_{j}\right), j=1, \ldots, n$ where polynomial $C(E)$ is defined by (3.6).

Systems (3.7)-(3.8) and (3.10)-(3.11) can be solved analytically by reducing them to Jacobi inversion problems. We will demonstrate the general method in the next section. These equations are also easily integrated numerically. Perhaps the best method for accomplishing numerical integration with the use of symplectic integrators. Such integrators preserve the Poincare invariants and are stable over a long period of time, see for example Channell and Scovel(1990).

\subsection{The Trace Formulas.}

The connection between the solutions $u$ and $v$ of the cKdV equation and the $\mu_{i}$ 's from (3.7),(3.8) is derived in Appendix $B$ and is given by

$$
\begin{aligned}
& u=2 \kappa \sum_{i=1}^{n} \mu_{i}+2 \kappa K_{1}, \\
& v=-2 \kappa \sum_{i<j \leq n} \mu_{i} \mu_{j}+\frac{3}{4 \kappa} u^{2}-K_{1} u+K_{2},
\end{aligned}
$$


where

$$
\begin{aligned}
& K_{1}=-\frac{1}{2} \sum_{i=1}^{2 n+2} m_{i}, \\
& K_{2}=\kappa \sum_{i<j \leq 2 n+2} m_{i} m_{j}-\kappa K_{1}^{2} .
\end{aligned}
$$

Observe that the small parameter $K_{1}$ from (1.1) is zero if $\sum_{i=1}^{2 n+2} m_{i}=0$. The trace formulas for the cDym system are

$$
\begin{aligned}
u & =2 \sum_{i=1}^{n} \mu_{i}+2 K_{1}, \\
v & =-\frac{1}{4} u^{\prime \prime}-2 \sum_{1 \leq i<j \leq n} \mu_{i} \mu_{j}+\frac{3}{4} u^{2}-K_{1} u+K_{2} .
\end{aligned}
$$

\section{Classification of Limits of Periodic Solutions}

For the next three sections we seek various periodic (genus-one) solutions of the Boussinesq System. Therefore unless otherwise stated we assume $r=0$ from (2.10) and $\kappa=1$ from (2.6). We first obtain the periodic traveling-wave solutions and then show that they are equivalent to solutions obtained in terms of a $\mu$ variable in the case $n=1$. This provides a natural introduction to the algebraic geometric method. A one-soliton solution is then obtained by deforming the Riemann surface of the genus-one periodic solution. This method of first finding periodic/quasi-periodic solutions and then deforming the level set (Riemann surface) in the phase space to obtain soliton solutions will be utilized throughout this paper. (For details about general approach see amongst others Ablowitz and $\mathrm{Ma}(1981)$ and Alber and Alber(1985).) 


\subsection{Periodic Traveling-Wave Solutions}

Let $U=U(\zeta)$ and $W=W(\zeta)$ where $\zeta=x-c t$ so that the Boussinesq System becomes

$$
\begin{aligned}
-c U^{\prime}+W^{\prime}+U U^{\prime} & =\gamma U^{\prime}, \\
-c W^{\prime}+U^{\prime \prime \prime}+(W U)^{\prime} & =\gamma W^{\prime},
\end{aligned}
$$

where $W^{\prime}$ and $U^{\prime}$ denote differentiation with respect to $\zeta$. Then (4.1) gives

$$
W=\eta U-\frac{1}{2} U^{2}+\tau_{0}
$$

where $\eta=c+\gamma$ and $\tau_{0}$ is a constant of integration. Plugging this into (4.2) and integrating twice gives

$$
\frac{\tau_{0}-\eta^{2}}{2} U^{2}+\frac{\eta}{2} U^{3}+\frac{1}{2}\left(U^{\prime}\right)^{2}-\frac{1}{8} U^{4}=\tau_{1} U+\tau_{2}
$$

where $\tau_{1}, \tau_{2}$ are constants of integration. Writing this as an integral equation and taking a square root we obtain

$$
d \zeta= \pm \frac{d U}{\sqrt{C_{4}(U)}}
$$

where

$$
C_{4}(U)=\prod_{l=1}^{4}\left(U-m_{j}\right)=U^{4}-4 \eta U^{3}-4\left(\tau_{0}-\eta^{2}\right) U^{2}-8 \tau_{1} U-8 \tau_{2} .
$$

Notice that the right hand side of this differential equation is multi valued since it involves a square root. This is uniquely defined on a Riemann surface of genus 1 parametrized by a pair $(W, E)$ where

$$
W^{2}=C_{4}(E)=\prod_{l=1}^{4}\left(E-m_{j}\right) .
$$

One indicates one of two sheets of the Riemann surface by choosing a particular sign in front of the square root: $W= \pm \sqrt{C_{4}(E)}$. Therefore $U$ in (4.5) is considered on a particular sheet of the Riemann surface (4.6) and so meaningful integration can take place. 
The equation (4.5) can also be obtained from the $\mu$-equations (3.7) and (3.8) for $n=1$. Notice that the trace formula shows that $U$ and $\mu$ are linearly related so that one can substitute $\mu$ instead of $U$ in (4.5). Using $\mu$ in this case will make it consistent with the formulas in the case when $n=2$ to be described in the next section.

After integrating (4.5), the following Jacobi inversion problem is obtained

$$
\theta=x-c t+\theta_{0}=\frac{1}{2} \int_{\mu_{0}}^{\mu} \frac{d \mu}{\sqrt{\prod_{i=1}^{4}\left(\mu-m_{i}\right)}},
$$

As stated before, this is a typical Jacobi inversion problem (Mumford (1983)). This integral is inverted using Jacobi's elliptic functions,

$$
\frac{\sqrt{\left(m_{1}-m_{3}\right)\left(m_{2}-m_{4}\right)}}{2} \int_{\mu_{0}}^{\mu} \frac{d \mu}{\sqrt{\prod_{i=1}^{4}\left(\mu-m_{i}\right)}}=\int_{z_{0}}^{z} \frac{d z}{\sqrt{\left(1-z^{2}\right)\left(1-k^{2} z^{2}\right)}}
$$

where

$$
z^{2}=\frac{\left(m_{2}-m_{4}\right)\left(\mu-m_{1}\right)}{\left(m_{1}-m_{4}\right)\left(\mu-m_{2}\right)}, k^{2}=\frac{\left(m_{1}-m_{4}\right)\left(m_{2}-m_{3}\right)}{\left(m_{2}-m_{4}\right)\left(m_{1}-m_{3}\right)}
$$

Notice that (4.8) is an elliptic integral of the first kind (see Mumford (1983)). Therefore, $\mu$ is obtained explicitly and

$$
\mu(x, t)=\frac{m_{2}\left(m_{4}-m_{1}\right) \operatorname{sn}^{2}(k, \omega)+m_{1}\left(m_{2}-m_{4}\right)}{m_{2}-m_{4}+\left(m_{4}-m_{1}\right) \operatorname{sn}^{2}(k, \omega)}
$$

where $\operatorname{sn}(k, \omega)$ is the Jacobi sine function and $\omega=\left(x-c t+\theta_{0}\right) / \sqrt{\left(m_{1}-m_{3}\right)\left(m_{2}-m_{4}\right)}$. This function is plotted in Figure B.1.

Periodic solutions correspond to the case when all $m_{i}$ are distinct. In this case, each fixed point in the phase space repels the trajectories so that for any initial value, $\mu$ oscillates periodically between the two nearest points. Notice that $\mu(0)$ must be chosen so that the right hand side of (4.7) is real. Then the fixed points repel and $\mu$ remains real valued. The solution $\mu$ leads to solutions for $U$ and $W$ through the trace formulas. The shape of $U$ is essentially the same as $\mu$. The shape 
of $W$ is aperiodic and is discussed further in the next section. Equation (4.7) can be interpreted as defining an angle variable $\theta$ where $c$ is then the action variable. From (4.7) it follows that in terms of these variables the initial Hamiltonian flow linearizes. It also can be viewed as an Abel -Jacobi map from a hyperelliptic curve

$$
W^{2}=C(E)=2\left(\mu-m_{1}\right)\left(\mu-m_{2}\right)\left(\mu-m_{3}\right)\left(\mu-m_{4}\right)
$$

or in other words, a Riemann surface of genus one, onto the Jacobi variety: $J=[\mathbf{C} \mid w Z]$ where $\mathbf{C}$ is a complex plane and $w$ is the period lattice of the holomorphic differential from (4.7).

\subsection{One-Soliton Solution of Kaup Type}

To examine soliton solutions, one deforms the Riemann surface $W^{2}=C_{4}(E)$. (For details about soliton deformations see amongst others Ablowitz and $\mathrm{Ma}(1981)$ and Alber and Alber(1985).) Here we consider the limit $m_{1} \rightarrow m_{2} \rightarrow a$, i.e. where the 2 roots $m_{1}$ and $m_{2}$ coalesce into one point. As this limit is approached, a soliton solution is obtained as the period of the periodic solution increases to infinity. In this case $a$ is called a double point. This double point is an attractor in the phase space. Without loss of generality, we assume $a<m_{3}<m_{4}$. This assumption leads to the realization that the only pertinent solution is obtained when $a<\mu(0)<m_{3}$.

On the principle branch of the square root,

$$
\begin{aligned}
x-c t+\theta_{0} & =\frac{1}{2} \int_{\mu_{0}}^{\mu} \frac{d \mu}{(\mu-a) \sqrt{\left(\mu-m_{3}\right)\left(\mu-m_{4}\right)}}, \\
& =-\left[\left(a-m_{4}\right)\left(a-m_{3}\right)\right]^{-1 / 2} \operatorname{arctanh}\left(\sqrt{\frac{\left(a-m_{4}\right)\left(\mu-m_{3}\right)}{\left(a-m_{3}\right)\left(\mu-m_{4}\right)}}\right),
\end{aligned}
$$

so that

$$
\mu=\frac{m_{3}\left(m_{4}-a\right)+m_{4}\left(a-m_{3}\right) \tanh ^{2}(\omega)}{\left(m_{4}-a\right)+\left(a-m_{3}\right) \tanh ^{2}(\omega)}
$$


where $\omega=-\left(x-c t+\theta_{0}\right) \sqrt{\left(a-m_{4}\right)\left(a-m_{3}\right)}$ and $a, m_{3}, m_{4}$ are functions of $c$. Using the trace formulas (3.12)-(3.13) and the transformations (A.3)-(A.4), the exact formulas for $U$ and $W$ from the Boussinesq equation are obtained. For example,

$$
U=\frac{-4 a^{2}+\left(m_{3}-m_{4}\right)^{2}+2 a\left(m_{3}+m_{4}\right)+\left(m_{4}^{2}-m_{3}^{2}\right) \cosh (2 \omega)}{2 a-\left(m_{3}+m_{4}\right)+\left(m_{3}-m_{4}\right) \cosh (2 \omega)}
$$

Notice that $\mu$ has a shape similar to a $\mathrm{KdV}$ solitary wave. It has a peak at $m_{3}$ and approaches $m_{1}$ for large $|x|$. From this we conclude that $U$ is also shaped like a KdV soliton with a peak of height $4 a+2 m_{4}-2 m_{3}$ and for large $|x|$ approaches $2 m_{3}+2 m_{4}$. One might be surprised that the soliton does not approach 0 as $x \rightarrow \pm \infty$. But remember that this is the modified cKdV equation with $K_{1} \neq 0$. Howver we may choose the parameters so that the solution does approach zero. The solution is plotted in Figure B.2. This solution was first found by Kaup(1972) using the inverse scattering transform in case when $m_{3}=-m_{4}$ and $a=0$, hence $K_{1}=0$. One advantage of the approach used here is that $W$ is easily found using the trace formulas, and the relationship between $U$ and $W$ is seen explicitly.

\subsection{Solutions with Two Peaks.}

Observe next that since $n=1, W$ is only a quadratic in $U$. The polynomial

$$
W=-\frac{1}{2} U^{2}+\left(2 a+m_{3}+m_{4}\right) U-4 a m_{3}-4 a m_{4}+\left(m_{3}-m_{4}\right)^{2},
$$

obtained from the trace formula is a parabola in $U$ with vertex at $U=2 a+m_{3}+m_{4}$. Since $U$ has the shape of a solitary wave, $W$ has either one or two peaks depending on the parameters defining the vertex of $U$. (See Figure B.2). If $U$ is concave down, then $W$ is a double peaked soliton if $m_{3}+m_{4}<2 a$ and $3 m_{3}<2 a+m_{4}$. In the unperturbed case $(\gamma=0)$, these conditions reduce to $m_{3}<0<a$. Otherwise $W$ has a single peak. The concave up case is similar. 


\subsection{One Kink Solution}

The one-kink solutions were initially found by Alonso and Rues(1992) as the simplest solutions obtained using the bilinear formalism of the Kyoto school. Our method introduces an alternate description of soliton fusion and fission for $n=2$ and simplifies the computations.

To obtain the one-kink solutions, we deform the Riemann surface further by taking the limit $m_{3} \rightarrow m_{4}$, so that $m_{1}=m_{2}=a_{1}$ and $m_{3}=m_{4}=a_{2}$. Note that if $\kappa=-1$ this limit is not permitted for real valued $\mu$ in (3.7). However, for $\kappa=1$ the system is readily solved for real $\mu$ producing a special case of equation (4.5) where $\tau_{1}=0=\tau_{2}=\gamma$. After inverting, the angle variable is

$$
\theta=x-c t+\theta_{0}= \pm \int \frac{2 d U}{U(U-2 c)}=\mp \frac{2}{c} \operatorname{arctanh}\left(\frac{U-c}{c}\right)
$$

and we find using the trace formulas and the transformations obtained in Appendix $\mathrm{A}$ that

$$
\begin{aligned}
U & = \pm c \tanh \left(-\frac{c}{2}\left(x-c t+\theta_{0}\right)\right)+c_{2}, \\
W & = \pm \frac{c^{2}}{2} \operatorname{sech}^{2}\left(-\frac{c}{2}\left(x-c t+\theta_{0}\right)\right) .
\end{aligned}
$$

These functions are plotted in Figure B.3. Here $U$ is always a kink or anti-kink while $W$ is always similar to a typical KdV soliton. Observe that the speed of the soliton, $c^{2} / 2$ is exactly the amplitude of $W$ and is proportional to the square of the amplitude of the $U$ soliton. This connection between wave speed and amplitude is reminiscent of that found in the $\mathrm{KdV}$ equation.

\section{Classification of Limits of Genus-Two Solutions}

In this section genus-two quasi-periodic solutions are constructed. By deforming the Riemann surface (spectral polynomial), two-soliton solutions are obtained and several types of soliton-soliton interactions are described. 


\subsection{Quasi-periodic Genus-Two Solutions}

In the genus-two case $U$ is given by the trace formulas to be the sum of two periodic functions, $\mu_{1}$ and $\mu_{2}$, and a constant. In general the functions, $\mu_{1}$ and $\mu_{2}$, have noncommensurate periods, so that the solution $U$ is generically quasi-periodic and systems (3.7) and (3.8) are defined on the symmetric product of two copies of the Riemann surface (hyperelliptic curve) of genus two given by

$$
W^{2}=C_{6}(E),
$$

where

$$
C_{6}(E)=\kappa \prod_{l=1}^{6}\left(E-m_{j}\right)
$$

After reordering the equations, summing them up, and integrating, the following Jacobi inversion problem is obtained:

$$
\begin{aligned}
\theta_{1} & =\int_{\mu_{1}^{0}}^{\mu_{1}} \frac{d \mu_{1}}{\sqrt{C_{6}\left(\mu_{1}\right)}}+\int_{\mu_{2}^{0}}^{\mu_{2}} \frac{d \mu_{2}}{\sqrt{C_{6}\left(\mu_{2}\right)}}=2 x-2 a_{1} t+\theta_{1}^{0}, \\
\theta_{2} & =\int_{\mu_{1}^{0}}^{\mu_{1}} \frac{\mu_{1} d \mu_{1}}{\sqrt{C_{6}\left(\mu_{1}\right)}}+\int_{\mu_{2}^{0}}^{\mu_{2}} \frac{\mu_{2} d \mu_{2}}{\sqrt{C_{6}\left(\mu_{2}\right)}}=2 x-2 a_{2} t+\theta_{2}^{0} .
\end{aligned}
$$

Inverting the Abel-Jacobi map defined by (5.3)-(5.4) results in expressions for $\mu_{1}$ and $\mu_{2}$ in terms of Riemann $\theta$-functions. (For details about the Abel-Jacobi map see Mumford(1983), Matveev and Yavor(1979), and Ercolani and McKean (1990).)

Having derived the genus-two quasi-periodic solutions, several limiting cases will now be explored to introduce solitons. Below each distinct case is considered.

\subsection{One-Soliton Solution on a Quasi-periodic Background}

A one-soliton solution on a quasi-periodic background is obtained in the limit $m_{1} \rightarrow m_{2} \rightarrow a$. Just as in the one-soliton case, a soliton is created as the Riemann surface is manipulated by pinching 
two elements of the spectrum. However, in this case there are two $\mu$ variables and the orbit for only one $\mu$-variable is changed. The other $\mu$ variable remains periodic. The result is a solution with a Kaup type soliton on a quasi-periodic background, and it is plotted in Figure B.4. The problem of inversion may be written in the following way,

$$
\begin{aligned}
\theta_{1} & =\int_{\mu_{1}^{0}}^{\mu_{1}} \frac{d \mu_{1}}{\left(\mu_{1}-a\right) \sqrt{P_{4}\left(\mu_{1}\right)}}+\int_{\mu_{2}^{0}}^{\mu_{2}} \frac{d \mu_{2}}{\left(\mu_{2}-a\right) \sqrt{P_{4}\left(\mu_{2}\right)}}=2 t+\theta_{1}^{0}, \\
\theta_{2} & =\int_{\mu_{1}^{0}}^{\mu_{1}} \frac{d \mu_{1}}{\sqrt{P_{4}\left(\mu_{1}\right)}}+\int_{\mu_{2}^{0}}^{\mu_{2}} \frac{d \mu_{2}}{\sqrt{P_{4}\left(\mu_{2}\right)}}=2 x-2 a t+\theta_{2}^{0}
\end{aligned}
$$

where

$$
P_{4}(E)=\left(E-m_{3}\right)\left(E-m_{4}\right)\left(E-m_{5}\right)\left(E-m_{6}\right)
$$

(For details about inverting problems of this type see Alber and Fedorov(1999).)

\subsection{Two-Soliton Solutions of Kaup Type}

The two-soliton solution is obtained by piecewise pinching together two pairs of elements of the spectrum so that $m_{1} \rightarrow m_{2} \rightarrow a_{1}$ and $m_{3} \rightarrow m_{4} \rightarrow a_{2}$. Here there are two double points $a_{1}$ and $a_{2}$, as well as two remaining hyperelliptic points at $m_{5}$ and $m_{6}$. Choosing the initial data on the positive branch of the Riemann surface for both $\mu$ variables leads to the following problem of inversion

$$
\begin{aligned}
\theta_{1}= & \int_{\mu_{1}^{0}}^{\mu_{1}} \frac{d \mu_{1}}{\left(\mu_{1}-a_{2}\right) \sqrt{\left(\mu_{1}-m_{5}\right)\left(\mu_{1}-m_{6}\right)}} \\
& +\int_{\mu_{2}^{0}}^{\mu_{2}} \frac{d \mu_{2}}{\left(\mu_{2}-a_{2}\right) \sqrt{\left(\mu_{2}-m_{5}\right)\left(\mu_{2}-m_{6}\right)}}=2 x-2 a_{1} t+\theta_{1}^{0}, \\
\theta_{2}= & \int_{\mu_{1}^{0}}^{\mu_{1}} \frac{d \mu_{1}}{\left(\mu_{1}-a_{1}\right) \sqrt{\left(\mu_{1}-m_{5}\right)\left(\mu_{1}-m_{6}\right)}} \\
& +\int_{\mu_{2}^{0}}^{\mu_{2}} \frac{d \mu_{2}}{\left(\mu_{2}-a_{1}\right) \sqrt{\left(\mu_{2}-m_{5}\right)\left(\mu_{2}-m_{6}\right)}}=2 x-2 a_{2} t+\theta_{2}^{0} .
\end{aligned}
$$

This angle representation is similar to the one found in the case of the defocusing NLS equation. (See Alber and Marsden(1994).) 
Notice that the $\theta_{i}$ 's are essentially the sum of two Kaup type solitons as $t \rightarrow \pm \infty$. The integrals in system $(5.8),(5.9)$ may be evaluated to obtain the following nonlinear algebraic system of equations for the $\mu_{i}$ :

$$
\begin{aligned}
& \left(s_{1625}+s_{1526}\right)\left(s_{2625}+s_{2526}\right)=A_{1}\left(s_{1625}-s_{1526}\right)\left(s_{2625}-s_{2526}\right) \\
& \left(s_{1615}+s_{1516}\right)\left(s_{2615}+s_{2516}\right)=A_{2}\left(s_{1615}-s_{1516}\right)\left(s_{2615}-s_{1516}\right)
\end{aligned}
$$

where $s_{i j k l}=\sqrt{\left(\mu_{i}-m_{j}\right)\left(a_{k}-m_{l}\right)}$ and

$$
\begin{aligned}
& A_{1}=\exp \left[-\sqrt{\left(a_{2}-m_{5}\right)\left(a_{2}-m_{6}\right)}\left(2 x-2 a_{1} t+\theta_{1}^{0}\right)\right], \\
& A_{2}=\exp \left[-\sqrt{\left(a_{1}-m_{5}\right)\left(a_{1}-m_{6}\right)}\left(2 x-2 a_{2} t+\theta_{2}^{0}\right)\right] .
\end{aligned}
$$

\subsection{Phase Shift Formulas}

When two solitons interact, they normally re-emerge with their initial profile and velocity. However, they have shifted ahead or behind where they would have been had there been no interaction at all. The amount a soliton shifts is called its phase shift, and the integral equations (5.8), (5.9) can be used to compute it. Assume that $a_{1}>a_{2}$ and define

$$
M(x)=\sqrt{\frac{x-m_{5}}{x-m_{6}}} .
$$

The phase shifts for the systems (5.8)-(5.9) are calculated by using the following procedure. First, consider the reference frame where $\theta_{1}$ is a constant, that is $2 x-2 a_{1} t+\theta_{1}^{0}=\alpha_{1}$ for some constant $\alpha_{1}$. Then observe that $\theta_{2}$ can be written only as a function of $t$ and $\alpha_{1}$. Namely, $\theta_{2}=\alpha_{1}+2\left(a_{1}-\right.$ $\left.a_{2}\right) t+\theta_{2}^{0}-\theta_{1}^{0}$. Notice that the integrals on the left hand side of (5.8) are exactly the expression for single Kaup type solitons. Integrals of this form may be integrated as

$$
\int_{\mu_{2}^{0}}^{\mu_{2}} \frac{d \mu_{2}}{2\left(\mu_{2}-a_{1}\right) \sqrt{\left(\mu_{2}-m_{5}\right)\left(\mu_{2}-m_{6}\right)}}=\frac{1}{2 \sqrt{\left(a_{1}-m_{5}\right)\left(a_{1}-m_{6}\right)}} \log \left|\frac{\psi-M\left(a_{1}\right)}{\psi+M\left(a_{1}\right)}\right|,
$$


where for clarity we have defined $\psi^{2}=M\left(\mu_{2}\right)^{2}$. Then we see that in this frame when $t \rightarrow \infty$ the right side of (5.9) goes to infinity so that the left hand side must also grow without bound. On evaluation of the integrals we see that this can only happen when $\psi \rightarrow-M\left(a_{1}\right)$. Similarly when $t \rightarrow-\infty, \psi$ must approach $M\left(a_{1}\right)$. Substituting these values for $\mu_{2}$ in (5.9) gives asymptotics for

$\theta_{1}$ as $t \rightarrow \infty$ and as $t \rightarrow-\infty$, respectively. The phase shift for one of the solitons will be the difference between the behavior of $\theta_{1}$ at minus infinity and its behavior at plus infinity in the frame given by $\theta_{1}=\alpha_{1}$. In this way we obtain the shift between phases before and after interaction for $\theta_{1}$ to be

$$
\Delta_{1}=\frac{1}{\sqrt{\left(a_{2}-m_{5}\right)\left(a_{2}-m_{6}\right)}} \log \left|\frac{M\left(a_{2}\right)+M\left(a_{1}\right)}{M\left(a_{2}\right)-M\left(a_{1}\right)}\right| .
$$

Similarly the phase shift for $\mu_{2}$ along $\theta_{2}=\alpha_{2}$ is computed to be

$$
\Delta_{2}=\frac{1}{\sqrt{\left(a_{1}-m_{5}\right)\left(a_{1}-m_{6}\right)}} \log \left|\frac{M\left(a_{1}\right)+M\left(a_{2}\right)}{M\left(a_{1}\right)-M\left(a_{2}\right)}\right| .
$$

These formulas were initially obtained by Matveev and Yavor(1979) by studying asymptotics of singular $\theta$-functions.

\subsection{Kink-Anitkink Interaction Solutions}

The interaction of kink and antikink solutions will now be considered. The two-kink solutions are constructed by taking the limit of the spectral parameters so that $m_{1} \rightarrow m_{2} \rightarrow a_{1}, m_{3} \rightarrow m_{4} \rightarrow a_{2}$, and $m_{5} \rightarrow m_{6} \rightarrow a_{3}$. We examine both the case when the $\mu_{i}$ 's are both initially on the positive branch of the Riemann surface and the case when only one of the $\mu_{i}$ 's is initially on the positive branch and the other is on the negative branch. In contrast to the KdV equation, this difference in the initial conditions produces qualitatively different solutions. This difference arises because the $\mathrm{KdV}$ and $\mathrm{cKdV}$ equations contain at least one hyperelliptic branch point, while all of the fixed points are double points for the two-kink solutions. 


\subsection{Initial values of $\mu_{1}$ and $\mu_{2}$ are chosen on the positive branches of the Rie- mann surface.}

In this situation, the angle variables are computed to be

$$
\begin{aligned}
\theta_{1}= & +\int_{\mu_{1}^{0}}^{\mu_{1}} \frac{d \mu_{1}}{\left(\mu_{1}-a_{2}\right)\left(\mu_{1}-a_{3}\right)} \\
& +\int_{\mu_{2}^{0}}^{\mu_{2}} \frac{d \mu_{2}}{\left(\mu_{2}-a_{2}\right)\left(\mu_{2}-a_{3}\right)}=2 x-2 a_{1} t+\theta_{1}^{0}, \\
\theta_{2}= & +\int_{\mu_{1}^{0}}^{\mu_{1}} \frac{d \mu_{1}}{\left(\mu_{1}-a_{1}\right)\left(\mu_{1}-a_{3}\right)} \\
& +\int_{\mu_{2}^{0}}^{\mu_{2}} \frac{d \mu_{2}}{\left(\mu_{2}-a_{1}\right)\left(\mu_{2}-a_{3}\right)}=2 x-2 a_{2} t+\theta_{2}^{0} .
\end{aligned}
$$

These integrals are tractable, and the symmetric polynomials $\mu_{1} \mu_{2}$ and $\mu_{1}+\mu_{2}$ which appear in the trace formulas, can be calculated explicitly from the resulting system

$$
\begin{aligned}
& \left(1-A_{1}\right) \mu_{1} \mu_{2}+\left(a_{3} A_{1}-a_{2}\right)\left(\mu_{1}+\mu_{2}\right)=A_{1} a_{3}^{2}-a_{2}^{2}, \\
& \left(1-A_{2}\right) \mu_{1} \mu_{2}+\left(a_{3} A_{2}-a_{1}\right)\left(\mu_{1}+\mu_{2}\right)=A_{2} a_{3}^{2}-a_{1}^{2},
\end{aligned}
$$

with

$$
\begin{aligned}
& A_{1}=\frac{\left(\mu_{1}^{0}-a_{2}\right)\left(\mu_{2}^{0}-a_{2}\right)}{\left(\mu_{1}^{0}-a_{3}\right)\left(\mu_{2}^{0}-a_{3}\right)} e^{2\left(a_{2}-a_{3}\right)\left(x-a_{1} t+\theta_{1}^{0}\right)} \\
& A_{2}=\frac{\left(\mu_{1}^{0}-a_{1}\right)\left(\mu_{2}^{0}-a_{1}\right)}{\left(\mu_{1}^{0}-a_{3}\right)\left(\mu_{2}^{0}-a_{3}\right)} e^{2\left(a_{1}-a_{3}\right)\left(x-a_{2} t+\theta_{1}^{0}\right)} .
\end{aligned}
$$

These calculations are carried out for the unperturbed Boussinesq equations $\left(K_{1}=0\right)$ in the next section and soliton fusion and fission are discussed.

\subsection{Soliton Fusion and Fission.}

Given this particular deformation of the Riemann surface, solitons can undergo fission or fusion. This interesting phenomenon occurs when two separate solitons enter an interaction but only one single soliton emerges from this interaction. Since all of the equations under study are invariant 
under the space-time inversion $(x \rightarrow-x, t \rightarrow-t)$, the reverse process of soliton fission may also occur where a single soliton breaks into two distinct solitons at some critical time. Observe that soliton fission can be interpreted as an infinite phase shift. This is because the solitons change their speed since when they fuse together. Therefore they will be infinitely far from where they would have been had there been no interaction. In this sense formulas (5.16)-(5.17) are still correct since in the limit $m_{5}=m_{6}=a_{3}, M(x)=1$ and so the formulas become singular.

For this discussion we assume that $\gamma=-2 \sum_{i=1}^{2 n+2} m_{i}=0$ although the general case is similar. Hence the system (5.20)-(5.21) can be solved for $\mu_{1}+\mu_{2}$ and is found to be

$$
U=-4\left(\mu_{1}+\mu_{2}\right)=-4 \frac{a_{1} f_{1}+a_{2} f_{2}+a_{3} f_{3}}{f_{1}+f_{2}+f_{3}}
$$

where

$$
\begin{aligned}
& f_{1}=\exp \left(2 a_{1} x+2 a_{1}^{2} t+\tilde{\theta_{1}}\right), \\
& f_{2}=\exp \left(2 a_{2} x+2 a_{2}^{2} t+\tilde{\theta_{2}}\right), \\
& f_{3}=\exp \left(2 a_{3} x+2 a_{3}^{2} t+\tilde{\theta_{3}}\right),
\end{aligned}
$$

and

$$
\begin{aligned}
& \tilde{\theta_{1}}=a_{1} \theta_{1}^{0}+\log \left[\left(a_{2}-a_{3}\right)\left(\mu_{1}^{0}-a_{1}\right)\left(\mu_{2}^{0}-a_{1}\right)\right], \\
& \tilde{\theta_{2}}=a_{2} \theta_{1}^{0}+\log \left[\left(a_{3}-a_{1}\right)\left(\mu_{1}^{0}-a_{2}\right)\left(\mu_{2}^{0}-a_{2}\right)\right], \\
& \tilde{\theta_{3}}=a_{3} \theta_{2}^{0}+\log \left[\left(a_{1}-a_{2}\right)\left(\mu_{1}^{0}-a_{3}\right)\left(\mu_{2}^{0}-a_{3}\right)\right] .
\end{aligned}
$$

The expression (5.24) coincides with a solution to Burger's equation which describes a confluence of shocks (see Whitham(1974)) and has the same form as a solution obtained by using the Hirota method. This expression is now analyzed to see why it represents soliton fusion. Suppose $a_{1}<$ $0<a_{2}<a_{3}$ and consider $t=0$. We claim that at this instant (5.24) is a two-tiered kink (sum of two kinks), see Figure B.8. To see why this is the case, consider $2 x<\left(\tilde{\theta_{2}}-\tilde{\theta_{1}}\right) /\left(a_{1}-a_{2}\right)$. For 
these values of $x, f_{1}$ is the largest of the terms $f_{1}, f_{2}$, and $f_{3}$. In fact as $x \rightarrow-\infty$, the terms $f_{2}, f_{3}$ are negligible in comparison to $f_{1}$. So for these values of $x, \mu_{1}+\mu_{2}$ is nearly constant and is approximately $a_{1}$. Similarly for $\left(\tilde{\theta_{2}}-\tilde{\theta_{1}}\right) /\left(a_{1}-a_{2}\right)<2 x<\left(\tilde{\theta_{3}}-\tilde{\theta_{2}}\right) /\left(a_{2}-a_{3}\right), f_{2}$ is the dominant term and $\mu_{1}+\mu_{2} \approx a_{2}$. For the remaining values of $x, f_{3}$ dominates so $\mu_{1}+\mu_{2} \approx a_{3}$.

We now apply this analysis for arbitrary $t$. In this manner we get that $U$ is essentially constant in three regions, $D_{1}, D_{2}$ and $D_{3}$, of the $(x, t)$ plane as seen in Figure B.7. When $t$ is sufficiently small, these regions are bounded by the points where the functions $f_{1}=f_{2}$ and where $f_{2}=f_{3}$. At

$$
t^{*}=\frac{\left(a_{2}-a_{3}\right)\left(\tilde{\theta_{2}}-\tilde{\theta_{1}}\right)-\left(a_{1}-a_{2}\right)\left(\tilde{\theta_{3}}-\tilde{\theta_{2}}\right)}{2\left(a_{1}-a_{2}\right)\left(a_{2}-a_{3}\right)\left(a_{1}-a_{3}\right)},
$$

the functions $f_{1}=f_{2}=f_{3}$ for some $x^{*}$. From this instant on, $f_{2}$ is never the largest term and so for $t>t^{*}$, the plane is divided into two regions bounded by the points where $f_{1}=f_{3}$ as seen in Figure B.7. For more details on this analysis see Whitham's(1974) chapter on Burger's equation. As explained above, the regions $D_{i}$, which contain all the information regarding fission of the two-kink solution (times, speeds etc.), are derived from the parameterization of the lines $f_{1}=f_{2}$, $f_{2}=f_{3}$, and $f_{1}=f_{3}$. These lines are computed explicitly giving that $f_{1}=f_{2}$ along the line where $2 x=2 a_{3} t+\left(\tilde{\theta_{2}}-\tilde{\theta_{1}}\right) /\left(a_{1}-a_{2}\right), f_{2}=f_{3}$ along the line where $2 x=2 a_{1} t+\left(\tilde{\theta_{3}}-\tilde{\theta_{2}}\right) /\left(a_{2}-a_{3}\right)$ and $f_{1}=f_{3}$ along the lines where $2 x=2 a_{2} t+\left(\tilde{\theta_{3}}-\tilde{\theta_{1}}\right) /\left(a_{1}-a_{3}\right)$. From this it is possible to predict the times at which two solitons experience fission or fusion based solely on the initial phase and the values of the three spectrum points $a_{i}$. The speeds of the solitons are given by the slopes of the lines, and in this way a complete explanation of fission is given. Notice that the arguments are quite general and that this approach can be applied directly to the entire class of $N$-component systems. 


\subsection{Initial values of $\mu_{1}$ and $\mu_{2}$ are chosen on different branches of the Riemann surface.}

This case gives a similar problem of inversion as the fusion case, namely

$$
\begin{aligned}
\theta_{1}= & +\int_{\mu_{1}^{0}}^{\mu_{1}} \frac{d \mu_{1}}{\left(\mu_{1}-a_{2}\right)\left(\mu_{1}-a_{3}\right)} \\
& -\int_{\mu_{2}^{0}}^{\mu_{2}} \frac{d \mu_{2}}{\left(\mu_{2}-a_{2}\right)\left(\mu_{2}-a_{3}\right)}=2 x-2 a_{1} t+\theta_{1}^{0}, \\
\theta_{2}= & +\int_{\mu_{1}^{0}}^{\mu_{1}} \frac{d \mu_{1}}{\left(\mu_{1}-a_{1}\right)\left(\mu_{1}-a_{3}\right)} \\
& -\int_{\mu_{2}^{0}}^{\mu_{2}} \frac{d \mu_{2}}{\left(\mu_{2}-a_{1}\right)\left(\mu_{2}-a_{3}\right)}=2 x-2 a_{2} t+\theta_{2}^{0} .
\end{aligned}
$$

These integrals may be evaluated in terms of the log function and give rise to the following algebraic equations for the $\mu_{i}$ 's

$$
\begin{aligned}
& \frac{\left(\mu_{1}-a_{1}\right)\left(\mu_{2}-a_{3}\right)}{\left(\mu_{1}-a_{3}\right)\left(\mu_{2}-a_{1}\right)}=\frac{\left(\mu_{1}^{0}-a_{1}\right)\left(\mu_{2}^{0}-a_{3}\right)}{\left(\mu_{1}^{0}-a_{3}\right)\left(\mu_{2}^{0}-a_{1}\right)} \exp \left[\left(a_{1}-a_{3}\right)\left(2 x-2 a_{2} t+\theta_{2}^{0}\right)\right], \\
& \frac{\left(\mu_{1}-a_{2}\right)\left(\mu_{2}-a_{3}\right)}{\left(\mu_{1}-a_{3}\right)\left(\mu_{2}-a_{2}\right)}=\frac{\left(\mu_{1}^{0}-a_{2}\right)\left(\mu_{2}^{0}-a_{3}\right)}{\left(\mu_{1}^{0}-a_{3}\right)\left(\mu_{2}^{0}-a_{2}\right)} \exp \left[\left(a_{2}-a_{3}\right)\left(2 x-2 a_{1} t+\theta_{1}^{0}\right)\right] .
\end{aligned}
$$

This is a system from which the $\mu_{i}$ 's can be found explicitly. The solutions are plotted in Figure B.9

and the corresponding $U$ and $W$ are graphed in Figure B.10. By initially choosing different branches of this particular Riemann surface we see that the solitons 'change form', i.e. a kink changes to an antikink and vice versa. An analytic explanation is given in the next section. This was first observed in Alonso and Rues(1992) who noticed this phenomenon asymptotically. Using the algebraic geometric construction the finite-time interactions can be analyzed as well.

\subsection{Change of Form of Kinks to Antikinks.}

The solutions obtained from (5.34) can be put in the following form

$$
\begin{aligned}
& \mu_{1}=\frac{-a_{1} g_{1}-a_{2} g_{2}-a_{3} g_{3}}{g_{1}+g_{2}+g_{3}}, \\
& \mu_{2}=\frac{a_{1} h_{1}+a_{2} h_{2}+a_{3} h_{3}}{h_{1}+h_{2}+h_{3}}
\end{aligned}
$$


where

$$
\begin{aligned}
& g_{1}=\exp \left[-2 a_{1} x-2 a_{2} a_{3} t-a_{1} \theta_{2}^{0}+\log \frac{\left(a_{3}-a_{2}\right)\left(\mu_{1}^{0}-a_{1}\right)}{\mu_{2}^{0}-a_{1}}\right], \\
& g_{2}=\exp \left[-2 a_{2} x-2 a_{1} a_{3} t-a_{2} \theta_{1}^{0}+\log \frac{\left(a_{1}-a_{3}\right)\left(\mu_{1}^{0}-a_{2}\right)}{\mu_{2}^{0}-a_{2}}\right], \\
& g_{3}=\exp \left[-2 a_{3} x-2 a_{1} a_{2} t-a_{3}\left(\theta_{1}^{0}+\theta_{2}^{0}\right)+\log \frac{\left(a_{2}-a_{1}\right)\left(\mu_{1}^{0}-a_{3}\right)}{\mu_{2}^{0}-a_{3}}\right], \\
& h_{1}=\exp \left[2 a_{1} x+2 a_{2} a_{3} t+a_{1} \theta_{2}^{0}+\log \frac{\left(a_{3}-a_{2}\right)\left(\mu_{1}^{0}-a_{1}\right)}{\mu_{2}^{0}-a_{1}}\right], \\
& h_{2}=\exp \left[2 a_{2} x+2 a_{1} a_{3} t+a_{2} \theta_{1}^{0}+\log \frac{\left(a_{1}-a_{3}\right)\left(\mu_{1}^{0}-a_{2}\right)}{\mu_{2}^{0}-a_{2}}\right], \\
& h_{3}=\exp \left[2 a_{3} x+2 a_{1} a_{2} t+a_{3}\left(\theta_{1}^{0}+\theta_{2}^{0}\right)+\log \frac{\left(a_{2}-a_{1}\right)\left(\mu_{1}^{0}-a_{3}\right)}{\mu_{2}^{0}-a_{3}}\right] .
\end{aligned}
$$

Notice the similarity between the form of the solutions (5.36), (5.37) and the solution (5.24) for $U$ in the previous case. Since they have an identical form as that in (5.24), all the analysis from the last section applies and we conclude that both $\mu_{1}$ and $\mu_{2}$ are kinks which experience fission or fusion respectively. We find that as $t \rightarrow-\infty, \mu_{1}$ is an antikink and $\mu_{2}$ decomposes into two kinks. This implies that $-\frac{1}{4} U=\mu_{1}+\mu_{2}$ consists of two kinks and one antikink. As $t \rightarrow \infty, \mu_{1}$ fissions into two antikinks and the 2 kinks comprising $\mu_{2}$ fuse into one kink. Therefore $\mu_{1}+\mu_{2}$ is the sum of one kink and two antikinks. This explains the transformation of kinks to antikinks and vice versa. See Figure B.11 to see how these $\mu$ variables combine to form $U$. Of course the same analysis can be performed as above to see this analytically. This is the first time two-soliton solutions of this equation have been derived in this simple form.

\section{The SHG Equations}

Solutions of the cKdV may also be transformed into solutions of the SHG equation if $\kappa$ is chosen to be -1 . This is shown explicitly in Appendix A. The results from the previous sections can be viewed in the context of the SHG equations. First, if $\kappa=-1$, the initial conditions must be chosen 
differently or the $\mu_{i}$ 's will not be real valued. That is, the cases $\kappa=1$ and $\kappa=-1$ are dual to each other in the sense that in the process of the construction of the Riemann surface the cuts in the complex plane for the $\kappa=1$ case correspond to where real valued solutions lie in the $\kappa=-1$ case and vice versa. This is because the kappa appears under the square root in (3.7) so that real values of $\mu$ for $k a p p a=1$ correspond exactly to imaginary values of $\mu$ when $\kappa=-1$ and vice versa.

Another important difference is that no kink solutions exist for the SHG equations. When $\kappa=-1$ there is no way to deform the Riemann surface so that all points coalesce piecewise as required for kink solitons. Therefore there are always at least two hyperelliptic points left. This means that SHG solutions do not have a possibility of either fusing or fissioning and no change of form can occur.

The phase shift formulas for this system are very similar to those in the cKdV hierarchy with $\kappa=1$, namely

$$
\begin{aligned}
& \Delta_{1}=\frac{1}{\sqrt{-\left(a_{2}-m_{5}\right)\left(a_{2}-m_{6}\right)}} \log \left|\frac{M\left(a_{2}\right)+M\left(a_{1}\right)}{M\left(a_{2}\right)-M\left(a_{1}\right)}\right| \\
& \Delta_{2}=\frac{1}{\sqrt{-\left(a_{1}-m_{5}\right)\left(a_{1}-m_{6}\right)}} \log \left|\frac{M\left(a_{1}\right)+M\left(a_{2}\right)}{M\left(a_{1}\right)-M\left(a_{2}\right)}\right|
\end{aligned}
$$

This is the first time these formulas have been derived. It is remarkable that the formulas are so similar to those of the coupled KdV equation since they are not in the same hierarchy of equations the two equations are derived from two different potentials. The phase shift formulas show another strength of the algebraic geometric method since the details of the inverse scattering transform have not been completed at this time, see Khusnutdinova(1998). $u$ and $v$ are plotted in Figure B.12. These functions can be transformed into $q_{1}, q_{2}$ of the SHG by transformations in Appendix A.

\section{Modified Coupled Dym Equations}




\subsection{Genus-1 solutions for the cDym System}

\subsubsection{Periodic solutions.}

A periodic solution of the cDym equation is described by the following differential equation

$$
\frac{\sqrt{\mu} d \mu}{\sqrt{\prod_{i=1}^{4}\left(\mu-m_{i}\right)}}=d X,
$$

for particular choice of $m_{i}$ 's. Here $X=2 x-2 c t+\theta_{0}$ and integration is carried out on the Riemann

surface $W^{2}=\frac{C(E)}{E}$. This can be reduced to a standard form by introducing a new variable $Y$ by

$$
d Y=\frac{d X}{\sqrt{\mu}}
$$

After integration (7.1) becomes

$$
\int_{\mu_{0}}^{\mu} \frac{d \mu}{\sqrt{\prod_{i=1}^{4}\left(\mu-m_{i}\right)}}=Y
$$

Notice that this holomorphic differential is defined on a genus two Riemann surface. To invert this integral one has first to consider the following problem of inversion

$$
\begin{aligned}
& \int_{\mu_{1}^{0}}^{\mu_{1}} \frac{d \mu_{1}}{\sqrt{\mu_{1} C_{4}\left(\mu_{1}\right)}}+\int_{\mu_{2}^{0}}^{\mu_{2}} \frac{d \mu_{2}}{\sqrt{\mu_{2} C_{4}\left(\mu_{2}\right)}}=\theta_{1}^{0} \\
& \int_{\mu_{1}^{0}}^{\mu_{1}} \frac{\mu_{1} d \mu_{1}}{\sqrt{\mu_{1} C_{4}\left(\mu_{1}\right)}}+\int_{\mu_{2}^{0}}^{\mu_{2}} \frac{\mu_{2} d \mu_{2}}{\sqrt{\mu_{2} C_{4}\left(\mu_{2}\right)}}=X_{1}+\theta_{2}^{0}
\end{aligned}
$$

where $C_{4}(\mu)=\prod_{l=1}^{4}\left(\mu-m_{j}\right)$. One needs to rearrange integrals in such a way that to obtain $X_{1}$ on the right hand side of the first integral equation. This yields exact formulas for $\mu_{1}$ and $\mu_{2}$ in terms of Riemann $\theta$-functions. By fixing $\mu_{2}=m_{3}$ and writing $\mu=\mu_{1}$ we resolve the initial problem of inversion. (For details see Alber and Fedorov(1999).) 


\subsubsection{Kink solutions.}

Now consider a kink limit by setting $m_{1}, m_{2} \rightarrow a_{1}$ and $m_{3}, m_{4} \rightarrow a_{2}$ such that $a_{2}>a_{1}>0$. Integral (7.3) becomes

$$
\frac{d \mu}{\left(\mu-a_{1}\right)\left(\mu-a_{2}\right)}=d Y
$$

Observe that this is the same problem of inversion as in the cKdV case except that we have $Y$ instead of $X$ on the right hand side. After integrating we obtain

$$
\mu(Y)=\frac{a_{1}\left(\mu_{0}-a_{2}\right)-a_{2}\left(\mu_{0}-a_{1}\right) \exp \left(\left(a_{1}-a_{2}\right) Y\right)}{\left(\mu_{0}-a_{2}\right)-\left(\mu_{0}-a_{1}\right) \exp \left(\left(a_{1}-a_{2}\right) Y\right)} .
$$

This gives $\mu$ as a function of $Y$ and as in the cKdV case this is a kink. $X$ is defined in terms of $Y$ by (7.2) once we know $\mu(Y)$ from (7.7). The integration in (7.2) may be carried out explicitly. Note that $d X / d Y>0$ and so $X(Y)$ is always increasing. By the definition of $X$ it is also clear that the range of $X(Y)$ is all real numbers. Therefore the Inverse Function Theorem implies that an inverse function $Y=Y(X)$ exists for all values of $X$ and is monotonically decreasing. Therefore the graph of $\mu(X)=\mu(Y(X))$ will have a similar appearance to that of $\mu(Y)$, that is, it is also a kink.

After combining numerics for $Y(X)$ with the expression for $\mu$, a description for the kink of the cDym system is obtained, see Figure B.13.

\subsubsection{Cusp solution}

For this solution, the limit $m_{1}, m_{2} \rightarrow a_{1}$ and $m_{3}, m_{4} \rightarrow a_{2}$ such that $a_{2}<0<a_{1}$ is analyzed. The analysis is the same as in the kink case except that now $d X / d Y$ changes sign exactly once when the branch point $Y^{*}$ is crossed where $\mu\left(Y^{*}\right)=0$. In this case, $Y(X)$ has two branches begining at the hyperelliptic point $X^{*}$ where $Y\left(X^{*}\right)=Y^{*}$. Therefore $\mu(X)$ has two branches and reaches a cusp at the point $X^{*}$. See Figure B.14. 


\subsubsection{Peakon solution}

If the Camassa-Holm shallow water equation is an indication(1994), a peakon may develop in the limit $m_{1}, m_{2} \rightarrow a_{1}$ and $m_{3}, m_{4} \rightarrow a_{2}$ with $a_{2}=0<a_{1}$. The analysis is similar to that of the kink case, except now the range of $X(Y)$ is bounded above by some number $X^{*}$. This means that the inverse function $Y(X)$ is defined only for those $X<X^{*}$. But, it can be defined symmetrically, as if the integration were carried out on the negative branch of the square root, and this gives rise to a peakon solution. The difference between this and a cusp solution is that in the cusp,

$$
\frac{d Y}{d X}=\frac{1}{\sqrt{\mu}}
$$

is infinite at the branch point, while in the Peakon case $\mu(Y)>0$ for all $Y$ and so at the branch point the derivative is finite.

\subsection{Genus 2 solutions}

The algebraic geometric procedure outlined thus far in this paper can be used for other equations as well, even when other methods may fail. (For details see Alber and Fedorov (1999).) Using our experience with the cKdV system, the case when there are three double points will be considered. For the positive branch of the Riemann Surface $W^{2}=C(E) / E$, the problem of inversion can be written as

$$
\begin{gathered}
\frac{\mu_{1} d \mu_{1}}{2\left(\mu_{1}-a_{1}\right)\left(\mu_{1}-a_{2}\right)\left(\mu_{1}-a_{3}\right) \sqrt{\mu_{1}}}+\frac{\mu_{2} d \mu_{2}}{2\left(\mu_{2}-a_{1}\right)\left(\mu_{2}-a_{2}\right)\left(\mu_{2}-a_{3}\right) \sqrt{\mu_{2}}}=d t, \\
\frac{\mu_{1}^{2} d \mu_{1}}{2\left(\mu_{1}-a_{1}\right)\left(\mu_{1}-a_{2}\right)\left(\mu_{1}-a_{3}\right) \sqrt{\mu_{1}}}+\frac{\mu_{2}^{2} d \mu_{2}}{2\left(\mu_{2}-a_{1}\right)\left(\mu_{2}-a_{2}\right)\left(\mu_{2}-a_{3}\right) \sqrt{\mu_{2}}}=d x .
\end{gathered}
$$

This inversion problem is very similar to that in the cKdV case, with the exception of the poles present in the left hand side of the equation. This case is complicated by the fact that only differentials of the third kind appear having simple poles at $a_{1}, a_{2}, a_{3}$. Following the procedure 
outlined in Alber and Fedorov(1999), a third variable $y$ is introduced such that

$$
\frac{d \mu_{1}}{2\left(\mu_{1}-a_{1}\right)\left(\mu_{1}-a_{2}\right)\left(\mu_{1}-a_{3}\right) \sqrt{\mu_{1}}}+\frac{d \mu_{2}}{2\left(\mu_{2}-a_{1}\right)\left(\mu_{2}-a_{2}\right)\left(\mu_{2}-a_{3}\right) \sqrt{\mu_{2}}}=d y .
$$

Also the normalized differentials of the third kind are introduced

$$
\Omega_{i}=\frac{\alpha_{i} d \mu}{\left(\mu-\alpha_{i}^{2}\right) \sqrt{\mu}}, \quad i=1,2,3
$$

where $\alpha_{i}=\sqrt{a_{i}}$. Next, consider three points $z_{i}$ given by

$$
\sum_{j=1}^{3} \int_{P_{0}}^{P_{j}} \Omega_{i}=z_{i}, \quad i=1,2,3 .
$$

The $z_{i}$ are dependent on $x, t, y$ by

$$
\begin{aligned}
& z_{1}=2 \sqrt{a_{1}}\left[x-\left(a_{2}+a_{3}\right) t+a_{2} a_{3} y\right], \\
& z_{2}=2 \sqrt{a_{2}}\left[x-\left(a_{1}+a_{3}\right) t+a_{1} a_{3} y\right], \\
& z_{3}=2 \sqrt{a_{3}}\left[x-\left(a_{1}+a_{2}\right) t+a_{1} a_{2} y\right] .
\end{aligned}
$$

Integrating (7.13) and putting $\xi=\sqrt{\mu}$ gives

$$
\frac{\left(\xi_{1}-\alpha_{i}\right)\left(\xi_{2}-\alpha_{i}\right)\left(\xi_{3}-\alpha_{i}\right)}{\left(\xi_{1}+\alpha_{i}\right)\left(\xi_{2}+\alpha_{i}\right)\left(\xi_{3}+\alpha_{i}\right)}=e^{z_{i}}, \quad i=1,2,3
$$

This gives the following system for the symmetric polynomials in $\xi$.

$$
\left(\begin{array}{ccc}
1-e^{z_{1}} & -\alpha_{1}\left(1+e^{z_{1}}\right) & \alpha_{1}^{2}\left(1-e^{z_{1}}\right) \\
1-e^{z_{2}} & -\alpha_{2}\left(1+e^{z_{2}}\right) & \alpha_{2}^{2}\left(1-e^{z_{2}}\right) \\
1-e^{z_{3}} & -\alpha_{3}\left(1+e^{z_{3}}\right) & \alpha_{3}^{2}\left(1-e^{z_{3}}\right)
\end{array}\right)\left(\begin{array}{c}
\xi_{1} \xi_{2} \xi_{3} \\
\xi_{1} \xi_{2}+\xi_{2} \xi_{3}+\xi_{1} \xi_{3} \\
\xi_{1}+\xi_{2}+\xi_{3}
\end{array}\right)=\left(\begin{array}{c}
\alpha_{1}^{3}\left(1+e^{z_{1}}\right) \\
\alpha_{2}^{3}\left(1+e^{z_{2}}\right) \\
\alpha_{3}^{3}\left(1+e^{z_{3}}\right)
\end{array}\right)
$$

¿From this, the expression $\mu_{1}+\mu_{2}+\mu_{3}=\xi_{1}^{2}+\xi_{2}^{2}+\xi_{3}^{2}$ may be found as a function of $z_{1}, z_{2}, z_{3}$. The determinant of the matrix in the system must be zero to obtain nonzero solutions. This added equation gives $\mu_{3}$ in terms of $\mu_{1}$ and $\mu_{2}$. Then we can find $\mu_{1}+\mu_{2}$ as a function of $z_{1}, z_{2}$. Then this solution must be connected to the $(x, t)$ variables by using (7.14). 
Numerics are provided in Figures B.15 and B.16. As expected, the phenomena of change of form and fission occurs. A more detailed analysis of fission/fusion for the cDym case will be described in a forth coming paper.

\section{Acknowledgments}

The research of Mark Alber and Gregory Luther was partially supported by NSF grant DMS 9626672. Mark Alber would like to thank Peter Miller for a helpful discussion and bringing to his attention references Estevez et al.(1994) and Martinez Alonso and Medina Reus(1992).

\section{A Transformations to Related Equations}

In what follows we describe exact connections between solutions of the $\mathrm{cKdV}$ and the Kaup equations, Boussinesq systems and SHG system.

\section{A.1 Generalized Coupled KdV System}

For this system, assume that $\kappa=1$ and $r=0$. Observe that if we take $n=1, B$ takes the form of $B=E+b_{1}$. Furthermore, as in (B.1) we see that $b_{1}=-u / 2+K_{1}$. Collecting coefficients of order 1 and 0 respectively in (2.9) and substituting the value of $b_{1}$ shows that $u, v$ satisfy the modified cKdV system

$$
\begin{aligned}
& u_{t}=v^{\prime}-\frac{3}{2} u u^{\prime}+K_{1} u^{\prime}, \\
& v_{t}=\frac{1}{4} u^{\prime \prime \prime}-v u^{\prime}-\frac{1}{2} u v^{\prime}+K_{1} v^{\prime} .
\end{aligned}
$$

$K_{1}$ is a small parameter. When $K_{1}=0$ this is the cKdV. 


\section{A.2 Generalized Boussinesq System}

To connect $u$ and $v$ to the classical Boussinesq system the following change of variables must be made

$$
\begin{aligned}
& u(x, t)=-\frac{1}{2} U(X, T), \\
& v(x, t)=\frac{1}{16} U(X, T)^{2}-\frac{1}{4} W(X, T),
\end{aligned}
$$

where $X=x$ and $T=-t / 2$. Plugging in $u$ and $v$ from (A.3)-A.4 into (A.1)-(A.2) and defining $\gamma=-2 K_{1}$ we see that $U$ and $W$ satisfy

$$
\begin{aligned}
U_{T}+W_{X}+U U_{X} & =\gamma U_{X}, \\
W_{T}+U_{X X X}+(W U)_{X} & =\gamma W_{X} .
\end{aligned}
$$

Again $\gamma$ is a small parameter. When $\gamma=0$, we have exactly the Boussinesq System. Otherwise the system gives rise to a generalization of the Boussinesq system. Such a transformation can be found in Sattinger(1995) for example.

\section{A.3 Generalized Kaup Equations}

To connect $U(X, T)$ and $W(X, T)$ from above to $\pi(x, t)$ and $\phi(x, t)$ from the Kaup equations (1.7), 1.8) the following change of variables was suggested by $\operatorname{Kaup}(1972)$ :

$$
\begin{aligned}
U(X, T) & =\frac{\epsilon}{\beta} \phi_{x}(x, t) \\
W(X, T) & =\beta^{-2}[1-\epsilon \pi(x, t)]
\end{aligned}
$$

where $X=x, T=\beta t, \beta=\delta \sqrt{3} / \sqrt{1-3 \sigma}$, and $\alpha=\beta \gamma$. Then substituting this into (A.5)-(A.6) gives

$$
\begin{aligned}
\pi_{t} & =\phi_{x x}+\frac{1}{3}(1-3 \sigma) \delta^{2} \phi_{x x x x}-\epsilon\left(\phi_{x} \pi\right)_{x}+\alpha \pi_{x} \\
\pi & =\phi_{t}+\frac{1}{2} \epsilon \phi_{x}^{2}-\alpha \phi_{x}
\end{aligned}
$$


which is a perturbed Kaup equations and reduces identically to it when $\alpha=0$. Summarizing we see that every solution of the coupled KdV system yields, using the transformations stated, a solution of the Boussinesq and Kaup systems.

\section{A.4 The SHG System}

For the SHG system we choose $\kappa=-1$ in the potential (2.6), and define $w, \nu$ by the equations $u=w$, and $v=\nu_{x} / 2+\nu^{2} / 4$. Then the generating equation (3.1) becomes

$$
\begin{aligned}
E w_{t}+\frac{1}{2} \nu_{x t}+\frac{1}{2} \nu \nu_{t}= & -\frac{1}{2} B_{x x x}+2 B_{x} w E+B_{x} \nu_{x}+\frac{1}{2} B_{x} \nu^{2} \\
& -2 B_{x} E^{2}+B w_{x} E+\frac{1}{2} B \nu_{x x}+\frac{1}{2} B \nu \nu_{x} .
\end{aligned}
$$

Now we choose $B=b / E$, that is we choose $m=1, n=-1, b=b_{-2}$. Then (A.11) becomes

$$
\begin{aligned}
E w_{t}+\frac{1}{2} \nu_{x t}+\frac{1}{2} \nu \nu_{t}= & -\frac{1}{2 E} b_{x x x}+2 b_{x} w+\frac{1}{E} b_{x} \nu_{x}+\frac{1}{2 E} b_{x} \nu^{2} \\
& -2 b_{x} E+b w_{x}+\frac{1}{2 E} b \nu_{x x}+\frac{1}{2 E} b \nu \nu_{x}
\end{aligned}
$$

Collecting coefficients of $E$ gives

$$
b=-\frac{\eta}{2}
$$

where we define $\eta$ by $\eta_{x}=w_{t}$. Then collecting coefficients of orders zero and one respectively and substituting in the value for $b$ gives

$$
\begin{aligned}
\frac{1}{2} \nu_{x t}+\frac{1}{2} \nu \nu_{t} & =-\eta_{x} w-\frac{1}{2} \eta w_{x} \\
0 & =\frac{1}{4} \eta_{x x x}-\frac{1}{2} \eta_{x} \nu_{x}-\frac{1}{4} \eta_{x} \nu^{2}-\frac{1}{4} \eta \nu_{x x}-\frac{1}{4} \eta \nu \nu_{x} .
\end{aligned}
$$

A.15) may be integrated to get

$$
\left(\eta_{x}\right)_{x}=(\eta \nu) \nu+\eta \nu_{x}+\int \nu_{x}\left(\eta \nu-\eta_{x}\right) d x
$$


Notice that this equation is satisfied when $\eta_{x}=\eta \nu$. Next we define the new function $s$ by the relation $\nu_{t}=s^{-1}-\eta w$. Then plugging this into (A.14) gives

$$
-\frac{s_{x}}{s^{2}}-\eta_{x} w-\eta w_{x}+\frac{\nu}{s}-\eta \nu w=-2 \eta_{x} w-\eta w_{x} .
$$

Substituting $\eta_{x}=\eta \nu$ and canceling like terms yields $s_{x}=s \nu$. These two equations, along with the two we defined will determine the SHG system. Summarizing, we have

$$
\begin{aligned}
& \eta_{x}=\eta \nu=w_{t}, \\
& \nu_{t}=s^{-1}-\eta w, \\
& s_{x}=s \nu .
\end{aligned}
$$

Next define $Q=s^{-1}$ and $\phi_{t}=\eta$. This and $\eta_{x}=w_{t}$ implies that $w=\phi_{x}$. Therefore (A.18) becomes

$$
\begin{aligned}
\left(Q \phi_{t}\right)_{x} & =Q_{x} \phi_{t}+Q \phi_{x t}, \\
& =-\frac{s_{x}}{s^{2}} \eta+\frac{\eta_{x}}{s}, \\
& =\frac{1}{s}\left(\eta_{x}-\eta \nu\right)=0,
\end{aligned}
$$

and

$$
\begin{aligned}
(\ln Q)_{x t}-\phi_{x} \phi_{t} & =-(\ln s)_{x t}-\eta w, \\
& =-\left(\frac{s_{x}}{s}\right)_{t}-\eta w, \\
& =-\nu_{t}-\eta w, \\
& =-\left(s^{-1}-\eta w\right)-\eta w=-Q .
\end{aligned}
$$

Finally, substitute $q_{1}=(\sqrt{Q} / 2) \exp [i(\phi / 2)]$ and the real and imaginary parts of the following equation correspond to (A.21) and (A.22), so that

$$
q_{1 x t} q_{1}^{*}-q_{1 t}^{*} q_{1 x}=-2\left(q_{1} q_{1}^{*}\right)^{2}
$$


If we define $q_{2}=-\left[(\ln Q)_{x}+i \phi_{x}\right] \exp (i \phi) / 4$. Then $q_{1}$ and $q_{2}$ satisfy

$$
\begin{aligned}
& q_{1 x}=-2 q_{2} q_{1}^{*}, \\
& q_{2 t}=q_{1}^{2},
\end{aligned}
$$

which is exactly the SHG equation, and $q_{1}$ and $q_{2}$ are obtained from the $\mu$ variables through the relation

$$
\begin{aligned}
\phi_{x} & =u \\
Q & =\exp \left(-\frac{u_{t}}{\int u_{t} d x}\right) .
\end{aligned}
$$

The above transformations were inspired by Khusnutdinova(1998). Summarizing the above gives that solutions of the SHG system may be expressed as follows

$$
\begin{aligned}
q_{1} & =\frac{1}{2} \exp \left(\frac{1}{2} \int u_{t}\left(\int u_{t} d x\right)^{-1} d x\right) \exp \left(\frac{i}{2} \int u d x\right) \\
q_{2} & =\frac{1}{4}\left(\frac{d^{2}}{d x^{2}} \log \left(\int u_{t} d x\right)-i u\right) \exp \left(i \int u d x\right)
\end{aligned}
$$

Notice that the dependence on $v$ is implicit by the fact that $\nu=u_{t}\left(\int u_{t} d x\right)^{-1}$.

\section{B Trace Formulas}

\section{B.1 cKdV System}

In this section, the connection between $u, v$, and $\mu$ for $c \mathrm{KdV}$ is derived. This connection is called the trace formula for this system. Collect coefficients of $E^{n-r+2}$ in (3.1) to see that $2 \kappa b_{0}^{\prime}=0$ from which we assume that $b_{0}=1$. Now gather coefficients of order $n-r+1$ to arrive at

$$
b_{1}=-\frac{u}{2 \kappa}+K_{1} \text {, }
$$


for some constant of integration $K_{1}$. Next collect coefficients of order $2 n-2 r+1$ in (3.2) to see, along with (B.1), that

$$
K_{1}=-\frac{1}{2} \sum_{i=1}^{2 n+2} m_{i}
$$

(B.1) then yields the trace formula for $u$,

$$
u=2 \kappa \sum_{i=1}^{n} \mu_{i}-\kappa \sum_{i=1}^{2 n+2} m_{i} .
$$

Now we just need the trace formula for $v$. For this we collect coefficients of order $n-r$ in (3.1) to get

$$
0=2 \kappa b_{2}^{\prime}+2 b_{1}^{\prime} u+b_{1} u^{\prime}+v^{\prime}
$$

which gives upon substitution of $b_{1}$ from (B.1) that

$$
v^{\prime}=-2 \kappa b_{2}^{\prime}+\frac{3 u u^{\prime}}{2 \kappa}-K_{1} u^{\prime}
$$

or simply

$$
v=-2 \kappa b_{2}+\frac{3 u^{2}}{4 \kappa}-K_{1} u+K_{2}
$$

where $K_{2}$ is some constant and $K_{1}$ is the same as above. From the definition of $B(E)$ we see that

$$
b_{2}=\sum_{1 \leq i<j \leq n} \mu_{i} \mu_{j}
$$

All that remains is to find $K_{2}$. To derive this we collect coefficients of order $2 n-2 r$ in (3.2). To simplify calculations let $c_{n}$ be the $(2 n-2 r)^{t h}$ coefficient of the polynomial $C(E)$. Then we see that

$$
2 \kappa\left(2 b_{2}+b_{1}^{2}\right)+2 u\left(2 b_{1}\right)+2 v=c_{n}
$$

or after substitution of the value of $b_{1}$ that

$$
4 \kappa b_{2}-\frac{3 u^{2}}{2 \kappa}+2 u K_{1}+2 \kappa K_{1}^{2}+2 v=c_{n} .
$$


Now solving for $K_{2}$ in (B.6) and substituting in $v$ from (B.9),

$$
\begin{aligned}
K_{2} & =v+2 \kappa b_{2}-\frac{3 u^{2}}{4 \kappa}+K_{1} u, \\
& =\left(\frac{c_{2}}{2}-2 \kappa b_{2}+\frac{3 u^{2}}{4 \kappa}-u K_{1}-\kappa K_{1}^{2}\right)+2 \kappa b_{2}-\frac{3 u^{2}}{4 \kappa}+K_{1} u, \\
& =\frac{c_{2}}{2}-\kappa K_{1}^{2} .
\end{aligned}
$$

Now from the definition of $C(E)$ we see that

$$
c_{n}=2 \kappa \sum_{1 \leq i<j \leq 2 n+2} m_{i} m_{j} .
$$

\section{B.2 cDym System}

In an analogous manner, we derive the trace formulas for the cDym system. The derivation of the trace formula for $u$ is identical to the $\mathrm{cKdV}$ case so that

$$
u=2 \sum_{i=1}^{n} \mu_{i}-\sum_{i=1}^{2 n+2} m_{i} .
$$

Only the trace formula for $v$ is different. For this we collect coefficients of order $n-r$ in (3.3) to get

$$
0=-\frac{b_{1}^{\prime \prime \prime}}{2}+2 b_{2}^{\prime}+2 b_{1}^{\prime} u+b_{1} u^{\prime}+v^{\prime}
$$

which, upon substitution of $b_{1}$ from (B.1) and integrating, gives

$$
v=-\frac{u^{\prime \prime}}{4}-2 b_{2}+\frac{3 u^{2}}{4}-K_{1} u+K_{2}
$$

where $K_{2}$ is some constant and $K_{1}$ is the same as in cKdV. From the definition of $B(E)$ we see that

$$
b_{2}=\sum_{1 \leq i<j \leq n} \mu_{i} \mu_{j}
$$


All that remains is to find $K_{2}$. To derive this we collect coefficients of order $2 n-2 r-1$ in (3.4). To simplify calculations let $c_{n-1}$ be the $(2 n-2 r-1)^{s t}$ coefficient of the polynomial $C(E)$. Then we see that

$$
-b_{1}^{\prime \prime}+2\left(2 b_{2}+b_{1}^{2}\right)+2 u\left(2 b_{1}\right)+2 v=c_{n-1},
$$

or after substitution of the value of $b_{1}$ that

$$
\frac{u^{\prime \prime}}{2}+4 b_{2}-\frac{3 u^{2}}{2}+2 u K_{1}+2 K_{1}^{2}+2 v=c_{n-1} .
$$

Now solving for $K_{2}$ in $(\overline{\mathrm{B} .16})$ and substituting in $v$ from $(\mathrm{B} .19)$ gives

$$
\begin{aligned}
K_{2} & =v+2 b_{2}-\frac{3 u^{2}}{4}+K_{1} u+\frac{u^{\prime \prime}}{4} \\
& =\left(\frac{c_{2}}{2}-2 b_{2}+\frac{3 u^{2}}{4}-u K_{1}-K_{1}^{2}-\frac{u^{\prime \prime}}{4}\right)+2 b_{2}-\frac{3 u^{2}}{4}+K_{1} u+\frac{u^{\prime \prime}}{4} \\
& =\frac{c_{n-1}}{2}-K_{1}^{2} .
\end{aligned}
$$

¿From the definition of $C(E)$ we see that

$$
c_{n-1}=2 \sum_{1 \leq i<j \leq 2 n+2} m_{i} m_{j}
$$




\section{Bibliography}

D. Mumford, Tata Lectures on Theta I and II, Progress in Math.28 and 43, (Birkhauser, Boston 1983).

Ercolani, N. and H. McKean, "Geometry of KdV(4). Abel sums, Jacobi variety, and theta function in the scattering case," Invent. Math 99, 483-544 (1990).

Ablowitz, M.J. and Y-C. Ma, "The Periodic Cubic Schrődinger Equation," Studies in Appl. Math. 65, 113-158 (1981).

Alber, M.S. and S.J. Alber, " Hamiltonian formalism for finite-zone solutions of integrable equations," C. R. Acad. Sci. Paris Sr. I Math. 301, 777-781 (1985).

Alber, M.S. and J.E. Marsden, "On Geometric Phases for Soliton Equations," Comm. Math. Phys. 149, 217-240 (1992).

Antonowicz, M. and A.P. Fordy, "A family of completely integrable multi-Hamiltonian systems", Phys. Lett. A 122, 95-99 (1987).

Antonowicz, M. and A.P. Fordy, "Coupled KdV equations with multi-Hamiltonian structures," Physica D 28, 345-357 (1987).

Antonowicz, M. and A.P. Fordy, "Coupled Harry Dym equations with multi-Hamiltonian structures," J. Phys. A 21, L269-L275 (1988).

Antonowicz, M. and A.P. Fordy, "Factorization of energy dependent Schrődinger operators: Miura maps and modified systems," Comm. Math. Phys. 124, 465-486 (1989).

Alber, M.S., G.G. Luther, and J.E. Marsden, "Energy Dependent Schrődinger Operators and Complex Hamiltonian Systems on Riemann Surfaces," Nonlinearity 10, 223-242 (1997). 
Channell, P.J. and C. Scovel, "Symplectic integration of Hamiltonian systems," Nonlinearity 3, $231-259$ (1990).

Channell, P.J. and C. Scovel, "An introduction to symplectic integrators," Fields Institute Communications 10, 45-58 (1996).

Alber, M.S., R. Camassa, D.D. Holm and J.E. Marsden, "The geometry of peaked solitons and billiard solutions of a class of integrable PDE's," Lett. Math. Phys. 32, 137-151 (1994).

Alber, M.S., R. Camassa, D.D. Holm and J.E. Marsden, "On Umbilic Geodesics and Soliton Solutions of Nonlinear PDE's," Proc. Roy. Soc. London Ser. A 450, 677-692 (1995).

Alber, M.S., R. Camassa, Yu.N. Fedorov, D.D. Holm and J.E. Marsden, "The geometry of new classes of weak billiard solutions of nonlinear PDE's," (preprint) (1999).

Alber, M.S. and Yu.N. Fedorov, "Algebraic Geometric Solutions for Nonlinear Evolution Equations and Flows on the Nonlinear Subvarieties of Jacobians," (preprint) (1999)

Whitham, G.B., Linear and Nonlinear Waves, (Pure and applied mathematics, John Wiley \& Sons, Inc. 1974).

Jaulent, M., "On an inverse scattering problem with an energy dependent potential," Ann. Inst. H. Poincare A 17, 363-372 (1972).

Jaulent, M. and C. Jean, "The inverse problem for the one-dimensional Schődinger operator with an energy dependent potential," Ann. Inst. H. Poincare A I, II25, 105-118, 119-137 (1976).

Kaup, D.J., "A Higher-Order Water-Wave Equation and the Method for Solving It," Prog. Theor. Phys. 54, 72-78, 396-408 (1975). 
Matveev, V.B. and M.I. Yavor, "Solutions presque periodiques et a $N$-solitons de léquation hydrodynamique non lineaire de Kaup," Ann. Inst. Henri Poincare: Sec. A 31, 25-41 (1979).

Sachs, R.L., "On the integrable variant of the Boussinesq system: Painlevé property, rational solutions, a related many-body system, and equivalence with the AKNS hierarchy," Physica D 30, 1-27 (1998).

Martinez Alonso, L. and E. Medina Reus, "Soliton interaction with change form in the classical Boussinesq system," Phys. Lett. A 167, 370-376 (1992).

Estevez, P.G., P.R. Gordoa, L. Martinez Alonso, and E. Medina Reus , "On the characterization of a new soliton sector in the classical Boussinesq system," Inverse Problems 10, L23-L27 (1994).

Khusnutdinova, K.R. and H. Steudel, "Second harmonic generation: Hamiltonian structures and particular solutions," J. Math. Phys. 39, 3754-3764 (1998).

Sattinger, David and Szmigielski, Jacek, "Energy dependent scattering theory," Differential and Integral Equations 8, 945-959 (1995).

Sattinger, David and Szmigielski, Jacek, "A Riemann Hilbert problem for an energy dependent Schrödinger operator," Inverse Problems 12, 1003-1025 (1996).

Ablowitz, M.J. and Segur, H. , Solitons and the Inverse Scattering Transform, (SIAM, Philadelphia, 1981).

Belokolos, E.D., A.I. Bobenko, V.Z. Enol'sii, A.R. Its, and V.B. Matveev, Algebro-Geometric Approach to Nonlinear Integrable Equations, (Springer-Verlag series in Nonlinear Dynamics, 1994). 
Ercolani, N. "Generalized Theta functions and homoclinic varieties," Proc. Symp. Pure Appl. Math. 49, 87-100 (1989).

Kupershmidt, B.A., "Mathematics of Dispersive Water Waves," Commun. Math. Phys. 99, 51-73 (1985). 


\section{Figures}

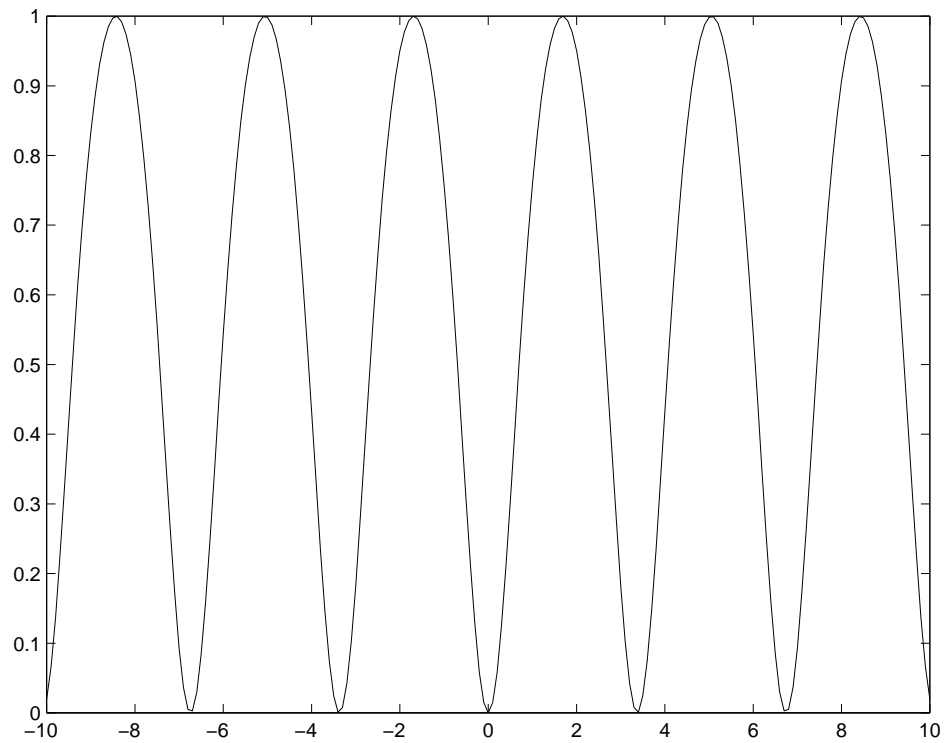

Figure B.1: Periodic $\mu$ solving (4.7) at $t=0$ in the genus- 1 case. This corresponds to distinct roots $m_{i}$ in (3.5). This plot uses $m_{1}=0, m_{2}=3, m_{3}=2, m_{4}=1$. From this function $\mu$, solutions $u, v$ of the cKdV equation may be obtained by (3.12),(3.13). Then $U, W$ from the Boussinesq equation may be found using (A.3) and (A.4). 


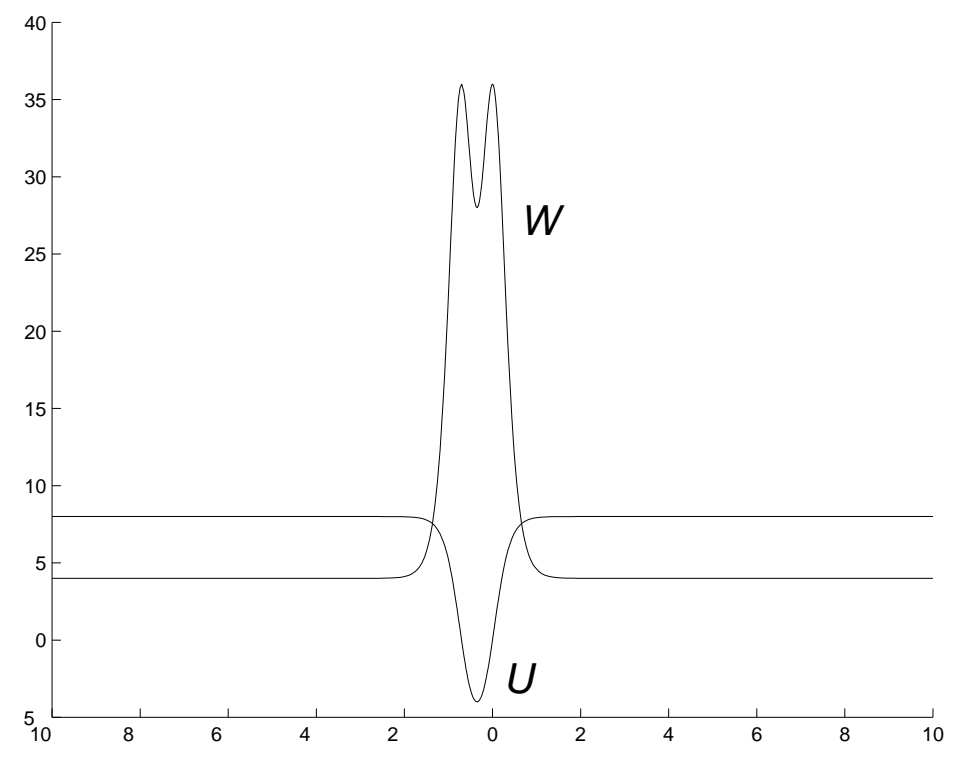

Figure B.2: Genus-one solutions $U$ and $W$ to the Boussinesq equation obtained from (4.15) using $a=-2, m_{3}=1, m_{4}=3$.

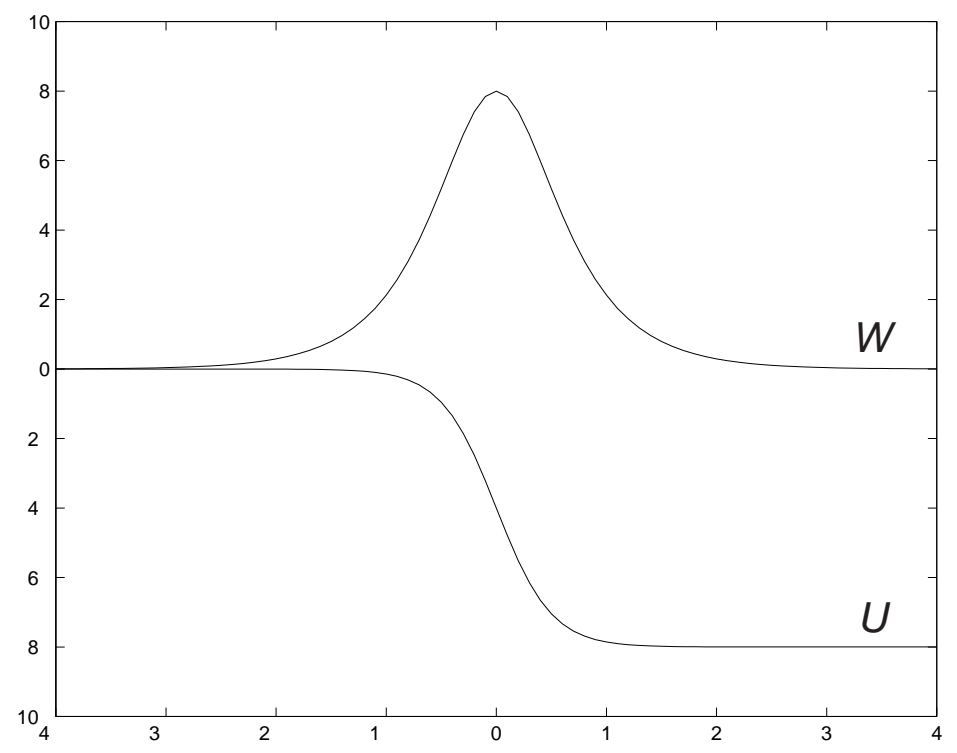

Figure B.3: $U$ and $W$ shown here are genus-one solutions to the Boussinesq equation for two double roots. $t=0, \theta_{0}=0, c=-4$. This graph highlights the simple relationship between $U$ and $W$ which will be important in understanding two soliton interaction. 


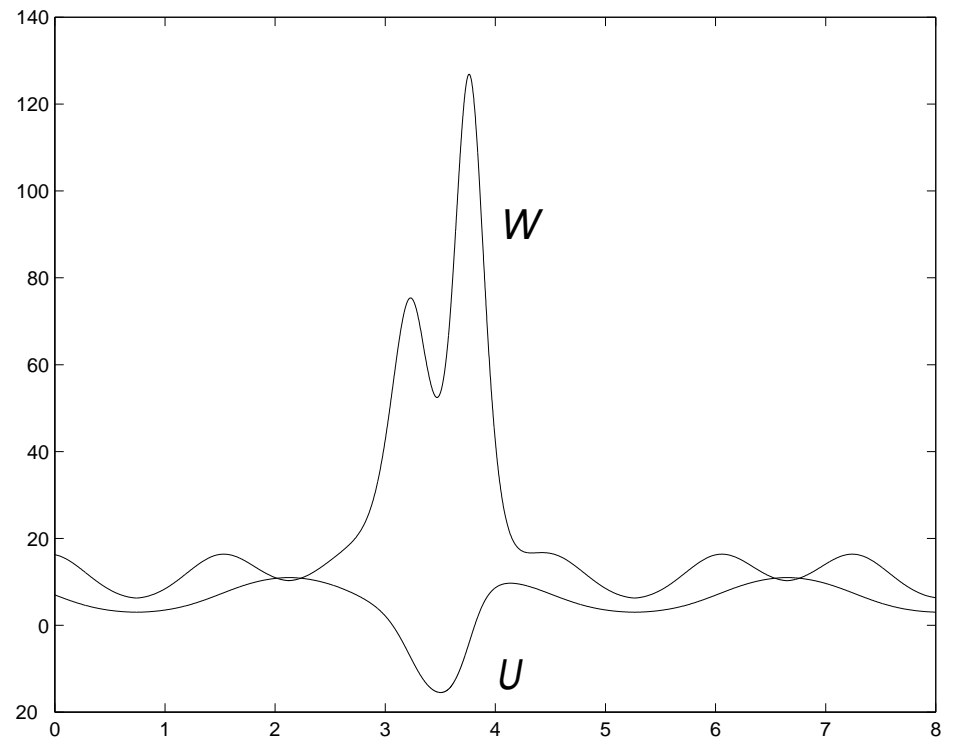

Figure B.4: Genus-two solutions $U$ and $W$ to the Boussinesq equation for one double root derived from (5.5), (5.6) and the trace formulas. This corresponds to a Kaup type soliton on a quasi-periodic background. Here $t=0, a=-5, m_{3}=0, m_{4}=1, m_{5}=3, m_{6}=3.5, \mu_{1}(0)=-4.99, \mu_{2}(0)=2$.
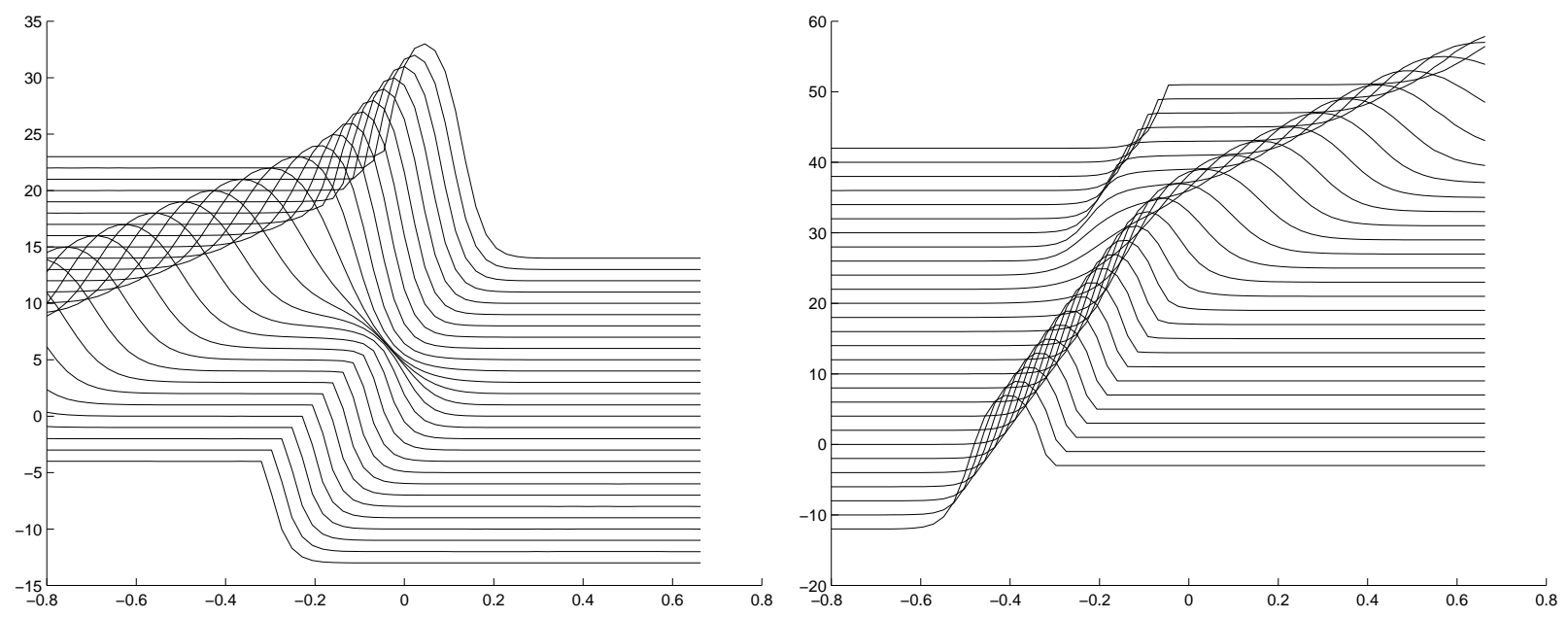

Figure B.5: These graphs show $\mu_{1}$ and $\mu_{2}$, genus-two solutions with two double roots, for $a 1=-5$, $a 2=-14, m_{5}=5, m_{6}=7.5, t=-.07$. This illustrates two Kaup type solitons. 

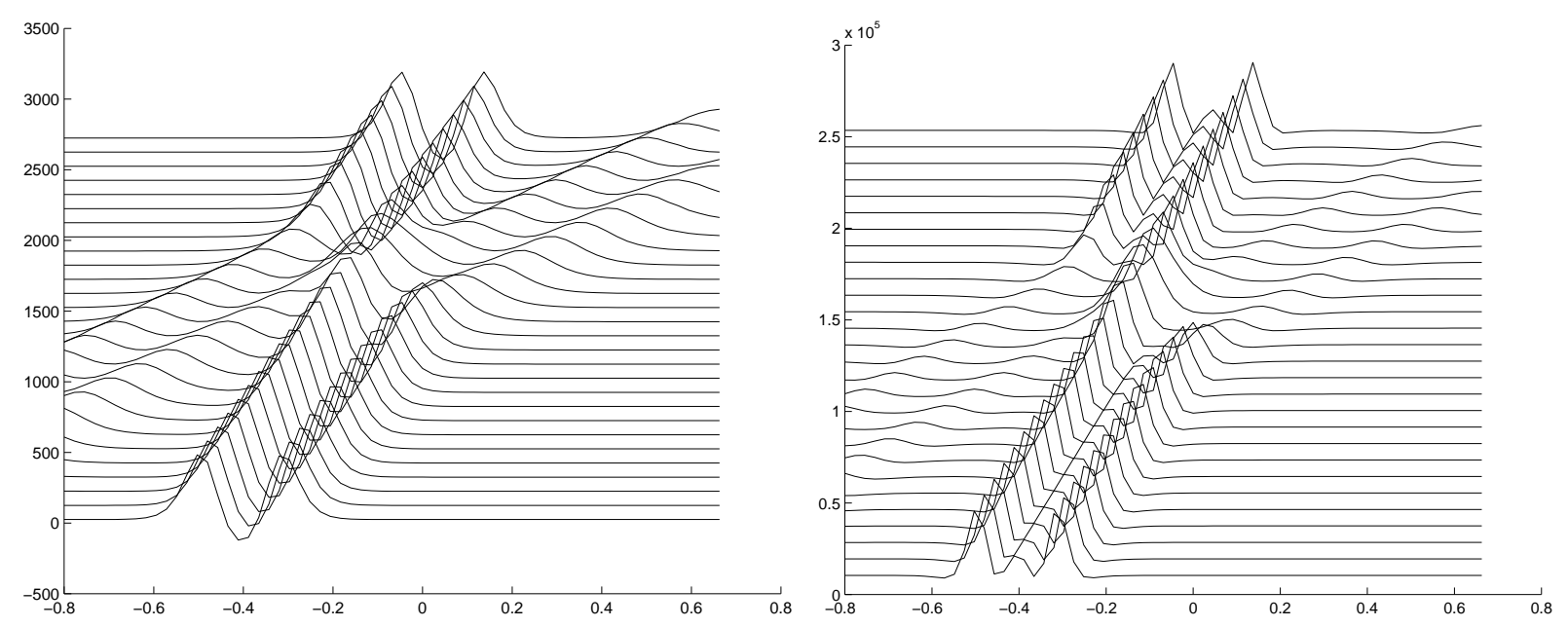

Figure B.6: This figure shows genus-two solutions $U$ and $W$ to the Boussinesq equation derived from (5.8), (5.9) and (A.3), (A.4). Here $a 1=-5, a 2=-14, m_{5}=5, m_{6}=7.5, t=-.07 \ldots .07$.

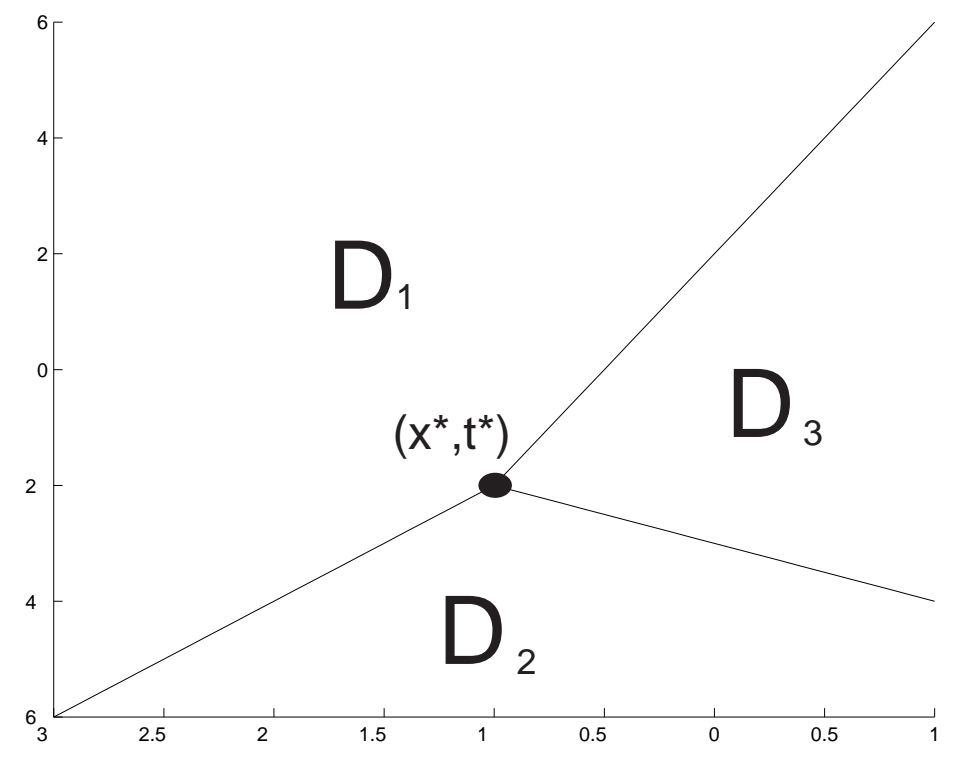

Figure B.7: Areas $D_{1}, D_{2}, D_{3}$ where away from the boundaries, $U$ from the Boussinesq equation has a constant height of $a_{1}, a_{2}, a_{3}$ respectively. Thus for a time $t<t^{*}, U$ is the sum of two kinks while for $t>t^{*}$, it is only one kink. This illustrates soliton fusion. 

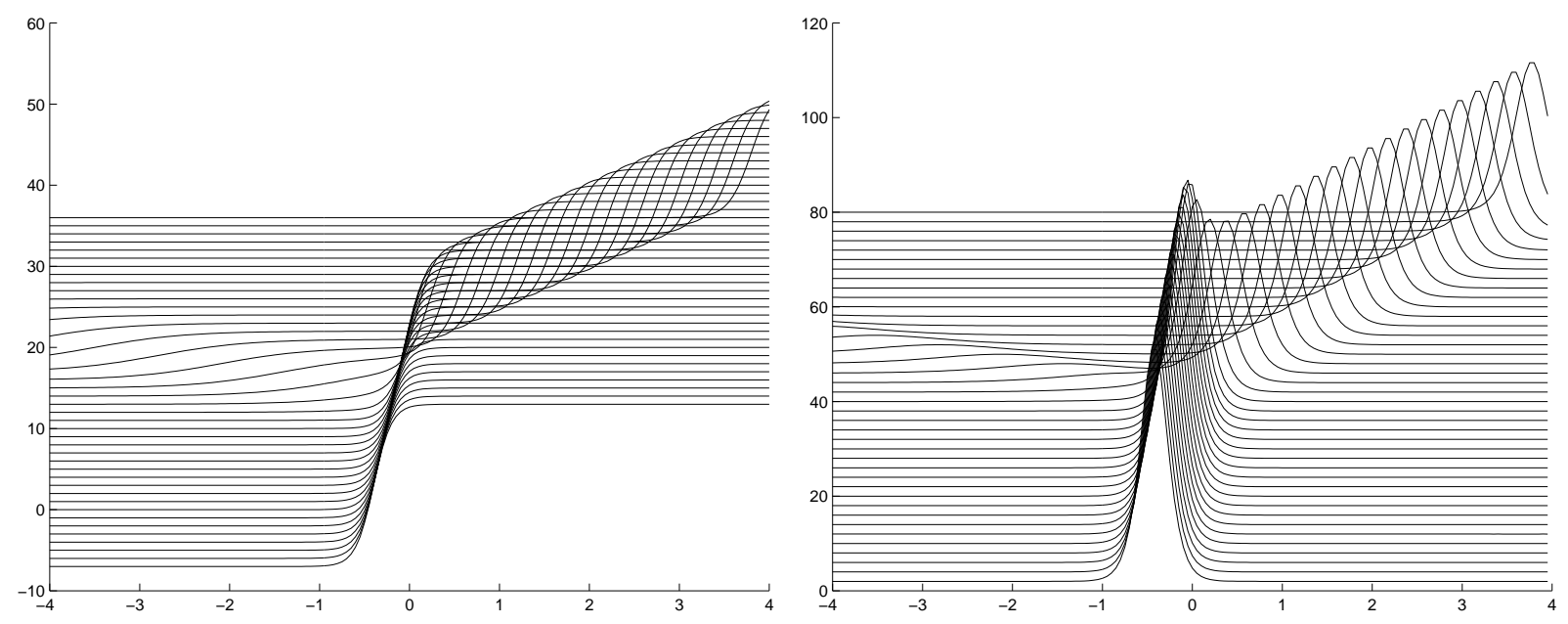

Figure B.8: Genus-two solutions $U$ and $W$ to the Boussinesq equation with two double roots. Here $a 1=-2, a 2=2, a_{3}=5, t=-.4 \ldots .4$. Both $\mu$ variables are initially chosen on the positive branch of the Riemann surface. Observe that each variable $U$ and $W$ undergoes soliton fission.
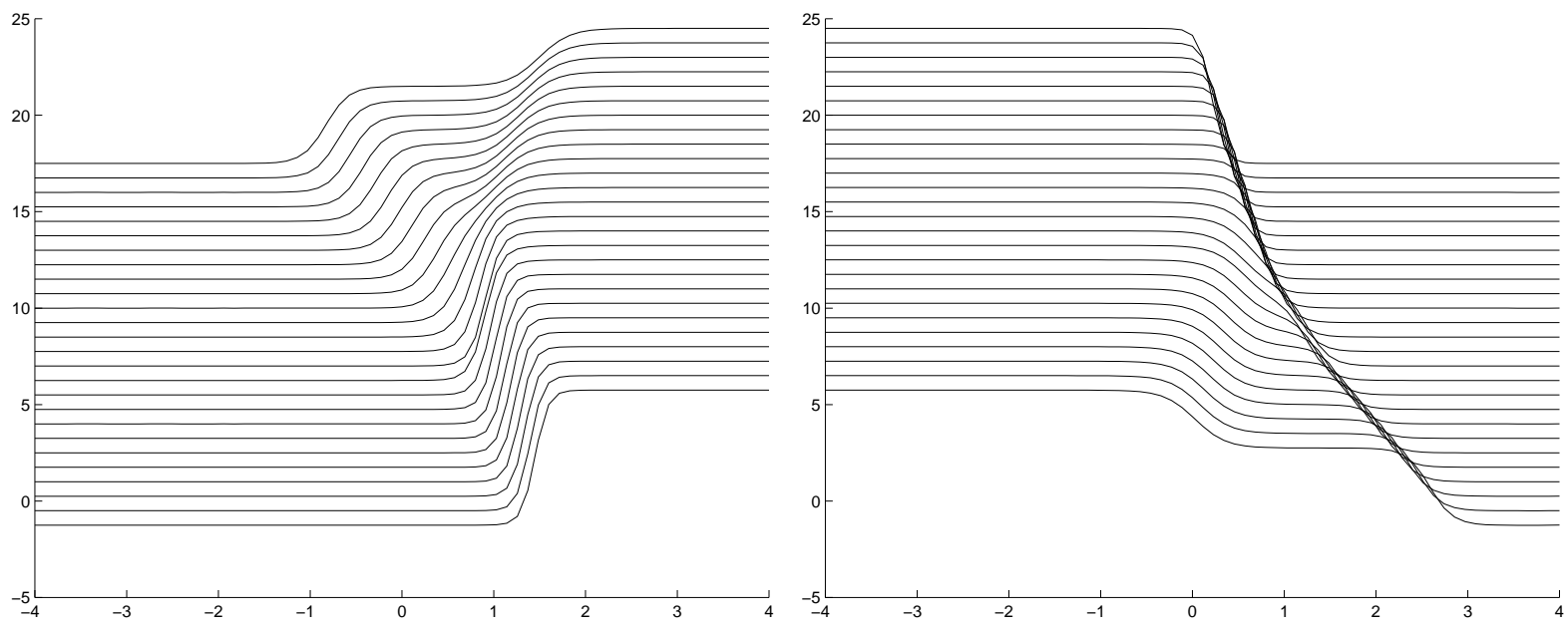

Figure B.9: Genus-two $\mu_{1}$ and $\mu_{2}$ from (5.36) and (5.37) illustrating when $\mu_{1}$ and $\mu_{2}$ are initially on different branches of the Riemann surface. The parameters chosen for these graphs are $a 1=-2$, $a 2=0, a 3=2, t=-2 \ldots 2$. Notice how similar these graphs are to the fission/fusion of $U$ in figure B.8 

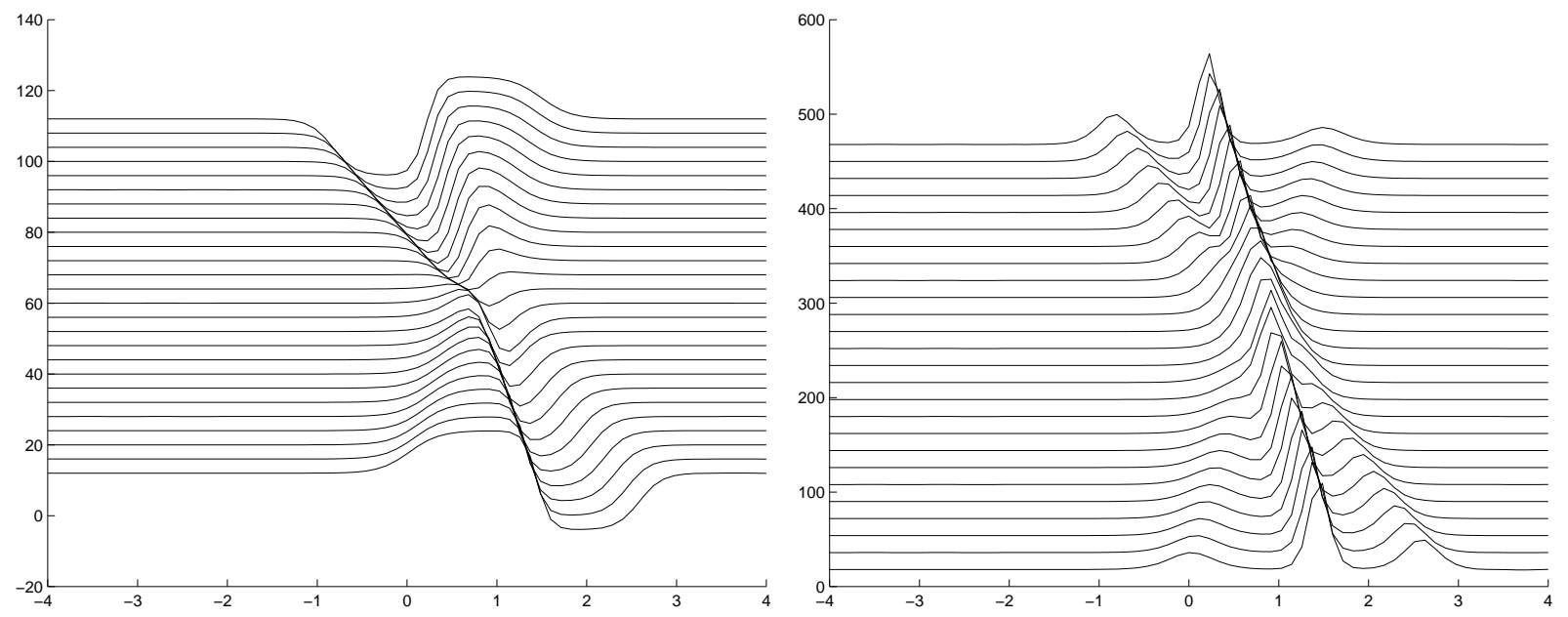

Figure B.10: Genus-two $U$ and $W$ from the Boussinesq equation derived from (5.36), (5.37) and (A.3), (A.4). Here $a 1=-2, a 2=2, a 3=5, t=-.4 \ldots 4$. Here the $\mu$ variables from (5.36) and (5.37) are chosen to be on the opposite branches of the Riemann surface. Notice the change of form in $U$ as kinks become antikinks and vice versa.

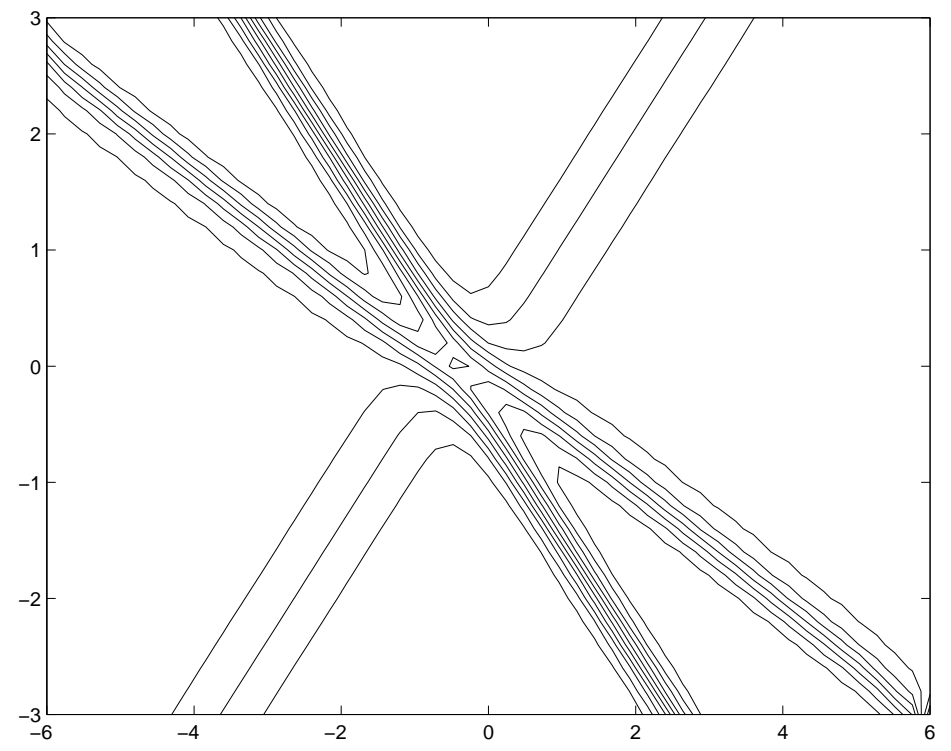

Figure B.11: A contour plot of a genus-two solution $U$ of the Boussinesq equation for the parameters $a 1=-1, a 2=1, a 3=2, t=-3 \ldots 3$. This shows how the $\mu$ variables combine to form different parts of the same kink. 


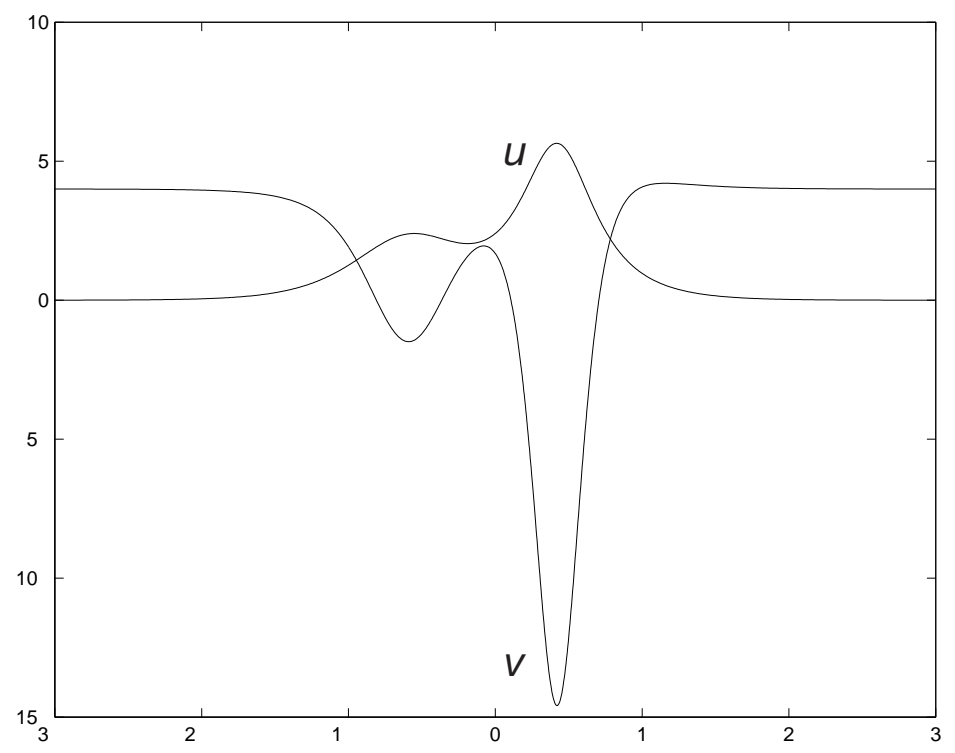

Figure B.12: A plot of $u$ and $v$, genus-two solutions derived from the $\mu$ variables in (5.8) and (5.9), except that $\kappa=-1$, using the trace formulas. These can be transformed into the SHG equation using transformations in appendix A. Here $(\kappa=-1)$ for $a 1=-1, a 2=1, m_{5}=2, m_{6}=-2, t=0$, and the $\mu$ variables are initially chosen on opposite branches of the Riemann surface.

Figure B.13: A genus-1 kink solution, $u(x, 0)$, for the coupled Dym equation. The parameters used were two double roots $a 1=1, a 2=2$, and $t=0$ 


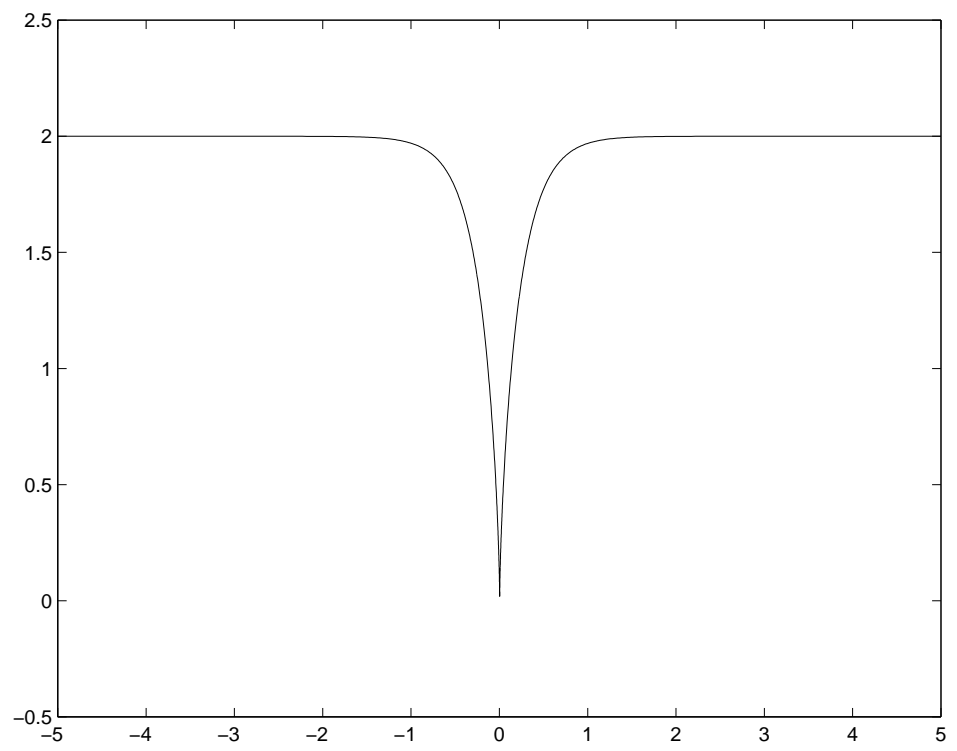

Figure B.14: A genus-1 cusp solution for the cDym equation. $u$ for two double roots $a 1=-1$, $a 2=1$, and $t=0$. 


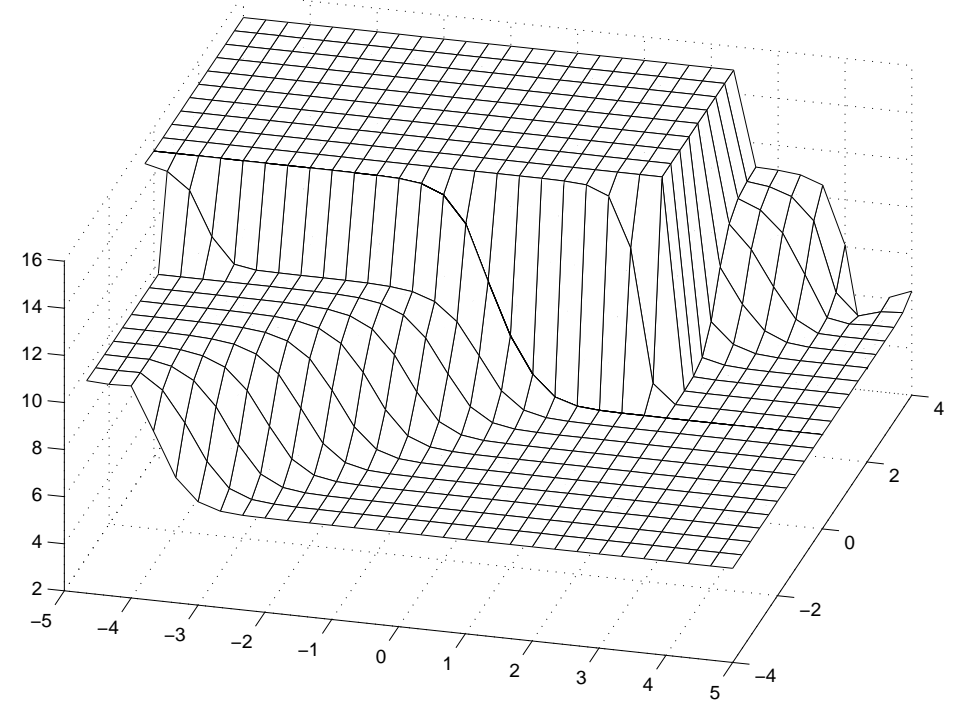

Figure B.15: Genus-two solution $u$ for the cDym equation with three double roots $a 1=.1, a 2=$ $.5, a_{3}=1, t=-12 \ldots 12$. Here the $\mu$ variables from which $u$ was derived were initially chosen to lie on the same branch of the Riemann surface. In this situation soliton fission occurs as in the ckdv case. 


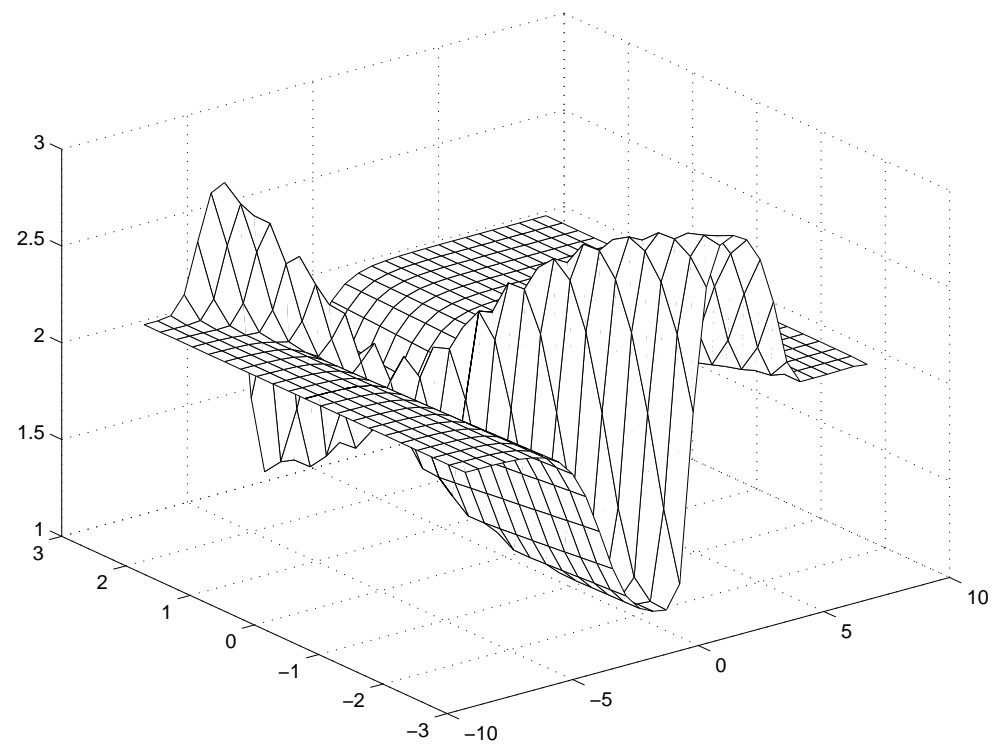

Figure B.16: Change of form for the Coupled Dym equation is seen for the genus-two solution $u$ with three double roots $a 1=1, a 2=5, a_{3}=10, t=-.3 \ldots 3$. The $\mu$ variables from which $u$ was derived were initially chosen on opposite branches of the Riemann surface. 